\title{
The orientation parameters and rotation curves of 15 spiral galaxies $\star \star \star \star$
}

\author{
A. M. Fridman ${ }^{1,2}$, V. L. Afanasiev ${ }^{3}$, S. N. Dodonov ${ }^{3}$, O. V. Khoruzhii ${ }^{1,4}$, A. V. Moiseev ${ }^{3,5}$, \\ O. K. Sil'chenko ${ }^{2,6}$, and A. V. Zasov ${ }^{2}$
}

${ }^{1}$ Institute of Astronomy of the Russian Academy of Science, 48, Pyatnitskaya St., Moscow 109017, Russia

2 Sternberg Astronomical Institute, Moscow State University, University prospect 13, Moscow 119992, Russia

3 Special Astrophysical Observatory, Nizhnij Arkhyz, Karachaevo-Cherkesia 357147, Russia

4 Troitsk Institute for Innovation and Thermonuclear Researches, Troitsk, Moscow reg. 142092, Russia

5 Guest investigator of the UK Astronomy Data Centre

${ }^{6}$ Isaac Newton Institute of Chile, Moscow Branch, Russia

Received 13 December 2002 / Accepted 1 September 2004

\begin{abstract}
We analyzed ionized gas motion and disk orientation parameters for 15 spiral galaxies. Their velocity fields were measured with the $\mathrm{H} \alpha$ emission line by using the Fabry-Perot interferometer at the $6 \mathrm{~m}$ telescope of SAO RAS. Special attention is paid to the problem of estimating the position angle of the major axis $\left(\mathrm{PA}_{0}\right)$ and the inclination $(i)$ of a disk, which strongly affect the derived circular rotation velocity. We discuss and compare different methods of obtaining these parameters from kinematic and photometric observations, taking into account the presence of regular velocity (brightness) perturbations caused by spiral density waves. It is shown that the commonly used method of tilted rings may lead to systematic errors in the estimation of orientation parameters (and hence of circular velocity) being applied to galaxies with an ordered spiral structure. Instead we recommend using an assumption of constancy of $i$ and $\mathrm{PA}_{0}$ along a radius, to estimate these parameters. For each galaxy of our sample we present monochromatic $\mathrm{H} \alpha$ - and continuum maps, velocity fields of ionized gas, and the mean rotation curves in the frame of a model of pure circular gas motion. Significant deviations from circular motion with amplitudes of several tens of $\mathrm{km} \mathrm{s}^{-1}$ (or higher) are found in almost all galaxies. The character and possible nature of the non-circular motion are briefly discussed.
\end{abstract}

Key words. galaxies: kinematics and dynamics - galaxies: spiral

\section{Introduction}

Most of the available data on the kinematics of ionized gas in disk galaxies were obtained with long-slit spectrographs, allowing us to construct mean rotation curves under an assumption of pure circular rotation. But the circular rotation model, being useful for determinations of the global properties of a galaxy, is only a zeroth order approximation to the real and rather complex dynamical picture.

Even if there is no active nucleus or tidal perturbation from a nearby companion to affect gas kinematics in a galaxy, the observed non-circular motion may have amplitudes up to $30-50 \mathrm{~km} \mathrm{~s}^{-1}$ (or even higher in some cases), being therefore supersonic. The nature of non-circular motion may be different. Small-scale gas motion is usually related to star formation sites

^ Based on observations collected with the $6 \mathrm{~m}$ telescope of the Special Astrophysical Observatory (SAO) of the Russian Academy of Sciences (RAS), operated under the financial support of the Science Department of Russia (registration number 01-43).

$\star \star$ Section 4 and Figs. 6-19 are only avalaible in electronic form at http://www. edpsciences.org
(HII complexes), whereas large-scale velocity perturbations are caused by wave spiral pattern and/or central oval (bar) impacts on the gaseous disk.

At the end of the 80s, a series of observations with a longslit spectrograph was undertaken at the $6 \mathrm{~m}$ telescope of the SAO RAS, which have revealed significant non-circular motion with amplitudes of $30-100 \mathrm{~km} \mathrm{~s}^{-1}$ in ordinary spiral galaxies (Afanasiev et al. 1988-1992). But the most complete information on the gas kinematics in galactic disks can be acquired only by using full 2D velocity fields which may be obtained in the optical spectral range by methods of panoramic (2D) spectroscopy. The method based on the Fabry-Perot interferometric observations was used by several groups of observers to get the velocity fields for large samples of different morphological types of galaxies (see f.e. Schommer et al. 1993; Amram et al. 1995). The largest project of this type is the GHASP survey, carried out at the Haute-Provence Observatory, which should provide a homogeneous sample of 2D velocity fields of about 200 spiral and irregular galaxies. This survey aimes to compare galaxies in different environments, different stages of evolution and at different redshifts (Garrido et al. 2002, 2003). Unlike the 
works mentioned above, the main goal of our project is to study the regular deviations of gas motion from circular rotation in spiral galaxies, which may be caused by spiral density waves in parallel with the routine task of obtaining the rotation curves and mass distributions. For this purpose we use the Scanning Fabry-Perot interferometer (IFP) of the $6 \mathrm{~m}$ telescope. We have obtained high-resolution line-of-sight velocity fields of the ionized gas over a large field of view (about 3') for 42 spiral galaxies ${ }^{1}$, both barred and non-barred, mostly of Sb-Sc types, by measuring the $\mathrm{H} \alpha$ (and in some cases also [NII]) emission line. This sample may be by no means considered as a complete one to some limiting magnitude or angular size. It just represents spiral galaxies of different types with the angular diameter of $2^{\prime}<D_{25}<6^{\prime}$ (for the most of them) having various types of spiral patterns - from certain grand-design to flocculent ones. The results of the velocity field analysis for some of the galaxies of our sample have already been published (Fridman et al. 1997, 1998, 1999, 2001a,b,c; Fridman \& Khoruzhii 2003). In this work we present the results of observations and the rotation curves for 15 galaxies of the sample. In parallel with the Doppler velocities measurements, brightness maps in $\mathrm{H} \alpha$ and near-H $\alpha$ continuum were also obtained for each galaxy.

In principle, the Doppler velocity field contains information both on the rotation and on non-circular gas motion, including the ordered wave motion related to a spiral density wave (if it exists in a chosen galaxy). The problem to be solved is the development of reliable methods which would enable us to extract and analyze these motions separately. Historically, the first attempts at such an approach were based on drawing a "mean" rotation curve, and on the consequent extraction of non-circular motion as residuals left after the subtraction of the circular rotation component from the observed velocity field (it was done for the first time by Warner et al. 1973, for M 33; and Pence 1981, for NGC 253). The attempts to relate the non-circular velocities found in this way to the observed spiral structure were unsuccessful, and this was treated by the authors as a failure to find non-circular motion of wave nature in these galaxies.

On the other hand, it was known from earlier papers that there exists a correlation between the velocity perturbations and the pattern of spiral structure in some galaxies (see for example Rubin et al. 1980, for optical rotation curves of galaxies or Rots \& Shane 1975, for a HI velocity field of M 81). If that is the case, can the negative result obtained for M33 and NGC 253 be accounted for by some peculiarities in these spiral galaxies? Indeed, although M 33 has two main spiral arms, it is really a multi-armed galaxy (Sandage \& Humphreus 1980). In turn, NGC 253 is a starburst galaxy with a rather high disk inclination, inappropriate for tracing spiral arms. However, later works by Sakhibov \& Smirnov $(1987,1989,1990)$ made it evident that the main problem of these early approaches is that independent determination of the rotation curve and of the residual velocities related to a density wave is not possible even for an "ideal" two-armed grand design galaxy. This conclusion was based on the analysis of the line-of-sight velocity fields of some galaxies by applying a Fourier-series expansion of

\footnotetext{
${ }^{1}$ For the full list, see http://www. sao.ru/hq/lsfvo/ vortex/vortex.html
}

velocity along the galactocentric azimuthal angle. The authors mentioned above showed that the even component of the first Fourier harmonic includes contributions from both the rotational velocity and the motion related to the density wave in the disk plane. Such interference makes it necessary to work out a special method to separate the circular and non-circular motion consistently. This method was proposed and described in detail in the papers by Lyakhovich et al. (1997), Fridman et al. (1997, 2001). These authors also showed that, in general, ignoring the influence of systematic (density-wave related) noncircular motion onto the line-of-sight velocities may prompt systematic errors in estimations of orientation parameters and lead to a wrong conclusion about their radial variations, even in the case of their constancy.

A similar problem appears when the photometric data is used to determine $i$ or $\mathrm{PA}_{0}$ of a disk. In this case the isophote distortion due to spiral arms may also mimic the radial variations of orientation parameters. To avoid this difficulty the outer disk isophotes are traditionally used where the contrast of spirals is usually low and may be neglected. But in some cases these regions may not be covered by the field of view, or are located too far from the galactic center so that their orientation differs from that of the inner disk (say, due to a disk warp). In this situation the correct estimation of orientation parameters needs a complex self-consistent analysis of brightness and/or of line-of-sight velocity distributions, taking into account both non-circular motion and brightness asymmetries, but this way is rather cumbersome for practical use. A simplified method should be chosen which may give an acceptable accuracy of orientation parameters. As it will be shown below, this problem is not trivial, and the use of different methods may lead to diverging results. Note that the accuracy of estimation of inclination and position angle of a galaxy influences not only the rotation curve obtained from observations. It may be a key factor which determines the possibility and the reliability of the evaluation of residual velocity field and of some refined parameters which, for example, may be used to distinguish chaotic and regular trajectories of gaseous clouds (Fridman et al. 2002).

Section 2 contains descriptions of our observations and data reduction procedures. In Sect. 3 we discuss various approaches to the determination of orientation parameters from the kinematic and photometric data and compare two independent methods, the combined use of which enables us to solve the problem with good accuracy. The parameters we found for 15 galaxies are used to obtain their velocity curves in a model of pure circular motion of gas. The discussion of individual galaxies is given in Sect. 4. Different types of non-circular gas motions are discussed in Sect. 5. The main results of this work are summarized in Sect. 6.

The detailed analysis of the systematic motions related to the density waves, based on the method described above, will be given in subsequent papers.

\section{Observations and data reduction}

The velocity fields of the chosen galaxies obtained in the $\mathrm{H} \alpha$ line cover significant parts of their discs, which enables us to use them to compare the estimates of the orientation 
Table 1. IFP parameters.

\begin{tabular}{lll}
\hline \hline & \multicolumn{2}{c}{ Fabry-Perot interferometers } \\
& FP235 & FP501 \\
\hline An order $($ at $\mathrm{H} \alpha$ line $)$ & 235 & 501 \\
Interfringe $(\Delta \lambda)$ & $28 \AA\left(1270 \mathrm{~km} \mathrm{~s}^{-1}\right)$ & $13 \AA\left(600 \mathrm{~km} \mathrm{~s}^{-1}\right)$ \\
Resolution $(\delta \lambda)$ & $3 \AA\left(130 \mathrm{~km} \mathrm{~s}^{-1}\right)$ & $1.2 \AA\left(130 \mathrm{~km} \mathrm{~s}^{-1}\right)$ \\
Spectral sampling: & & \\
$(24$-channel mode $)$ & $1.20 \AA\left(53 \mathrm{~km} \mathrm{~s}^{-1}\right)$ & $0.55 \AA\left(25 \mathrm{~km} \mathrm{~s}^{-1}\right)$ \\
$(32$-channel mode $)$ & $0.90 \AA\left(40 \mathrm{~km} \mathrm{~s}^{-1}\right)$ & $0.40 \AA\left(20 \mathrm{~km} \mathrm{~s}^{-1}\right)$ \\
\hline
\end{tabular}

parameters from both kinematic and photometric data and to obtain the rotation curves of the galaxies.

The observations were made in 1995-2000 with the Scanning Fabry-Perot Interferometer (IFP) attached to the reducer at the prime focus of the $6 \mathrm{~m}$ telescope of the SAO RAS; the equivalent focus distance of the system is $\mathrm{F} / 2.4$. The description of the interferometer can be found in Dodonov et al. (1995).

We limit the required spectral range by using a narrow interference filter with the $F W H M=10-20 \AA$ and a transmittance peak near a redshifted $\mathrm{H} \alpha$ line of a galaxy. When observing some galaxies (IC 1525, NGC 1084, etc.), the emission lines [NII] $\lambda$ 6548, 6583 from the neighbouring interference orders weakened by the wings of the filters also get into the passbands. Fortunately, when using any of our interferometers, the interference orders were overlaid in such a way that the gap between $\mathrm{H} \alpha$ and [NII] was not less than half of the full spectral range, so the presence of $[\mathrm{NII}]$ in the spectra did not prevent exact measurement of $\mathrm{H} \alpha$, excluding the case of the central part of NGC 5371 which is a LINER possessing rather broad emission lines.

During various observational runs we used two IFP by the Queensgate company which are operating in 235th and 501st interference orders (at a wavelength of $6562.78 \AA$ ) with the finesse of 8-12. The parameters of the interferometers are given in Table 1. The number of spectral channels (or steps of scanning) for various objects was 24 or 32 . Also, various types of panoramic detectors were used for different observational runs. In 1995-1996 we used a 2D photon counter (IPCS) with a format of $512 \times 512$ pixels, in $1997-$ a CCD with a format of $1040 \times 1160$ pixels, from 1998 to the present - the CCD TK1024 with a format of $1024 \times 1024$ pixels. In all cases the observations were made with binning of $2 \times 2$ pixels to increase the $\mathrm{S} / \mathrm{N}$ ratio and to economize the CCD readout time. The data are presented as "data cubes" consisting of 24 or 32 object images obtained with varying gaps between the interferometer plates.

For the different types of detectors we used different regimes of scanning. When observing with the IPCS, we accumulated an image in every channel during 15-20 s, and then the channel was switched forward. After passing through all the channels, the scanning cycle was repeated. This approach allowed us to overcome variations of atmospheric transparency and of seeing quality during total exposure. When observing with a CCD, each channel was exposed only once, but to properly take into account variations of atmospheric transparency, the odd channels $(1,3,5, \ldots)$ are exposed first, and then the even channels $(2,4,6, \ldots)$.

To correct spectra for the phase shift, we observe an emission line of the calibrating lamp. When observing with the IPCS, the calibration cubes were exposed before and after each object exposure; when observing with a CCD - at the beginning and at the end of the night. To correct the data for the modulation introduced by the order-separating filter and for the flat field, the telescope main mirror cup or the wall of the dome lit by a continuum light source are observed in every channel.

Reduction of the data obtained with the IPCS was performed in a standard way with the software ADHOC (Boulesteix 1993). It included a construction of the phase map by using the cube of the comparison spectrum, flat-fielding, phase-correcting the object cube (wavelength scale calibration), and sky spectrum subtraction based on analysis of detector areas free of the object emission and of its ghost images. To make an optimal filtration of the data and to increase the $\mathrm{S} / \mathrm{N}$ ratio in the regions with weak emission, we smoothed each spectrum in the spectral domain by a 1D Gaussian with $F W H M=2-3$ channels and in the space domain by a 2D Gaussian with $F W H M$ close to the seeing estimated from the measurements of nearby stars.

The primary reduction of the data obtained with CCDs is performed with the software developed by us and described in Moiseev (2002,b). It includes bias subtraction, flatfielding, cosmic hit cleaning, and mutual shifts of frames to overlay reference stars. The same stars are used to correct the datacube, if necessary, for the variations of atmospheric transparency and of seeing quality. Positions of the comparison spectrum line are determined by fitting its profile by a Gaussian or by a Lorenz profile; this modification has allowed us to achieve somewhat higher accuracy in the phase map construction with respect to the previous results obtained with ADHOC. After night-sky spectrum subtraction and correction for the phase shift, further reduction is performed by using the software ADHOC and a new version of this software for WINDOWS, ADHOCw (http: //www-obs. cnrs-mrs.fr/ADHOC/adhoc.html).

The journal of observations is given in Table 2, where for each galaxy we list its morphological type according to RC3, the date of the observations, the detector and the interferometer types which were used in each particular observation, pixel size after binning, total exposure time in each channel, seeing quality, and spatial resolution after all smoothing procedures estimated by using completely reduced star images in the cubes.

For each galaxy we have constructed a velocity field of the ionized gas by measuring barycenters of the $\mathrm{H} \alpha$ emission line in each spectrum (for one-component line profiles), as well as monochromatic images in $\mathrm{H} \alpha$ and red continuum images in the narrow passband near $\mathrm{H} \alpha$. If the emission-line profile is symmetric, the accuracy of the velocity measurements is restricted only by the accuracy of phase map constructing and is about $10-15 \mathrm{~km} \mathrm{~s}^{-1}$ for the observations with the IPCS and about $5 \mathrm{~km} \mathrm{~s}^{-1}$ for the CCD data. However in some cases (see below) the emission lines are clearly double-humped or possess strongly asymmetric profiles which is evidence for the 
Table 2. Log of IFP-observations.

\begin{tabular}{|c|c|c|c|c|c|c|c|c|c|}
\hline Name & & Type(RC3) & Date & Detector & IFP & $\overline{\text { px size }}$ & $\overline{T_{\text {exp }}, \mathrm{s}}$ & Seeing & Beam \\
\hline $\mathrm{IC}$ & 1525 & $\mathrm{SBb}$ & 04 Dec. 99 & CCD & FP235 & 0.68 & $24 \times 180$ & $2 . .5$ & $3 . \prime 2$ \\
\hline NGC & 23 & $\mathrm{SB}(\mathrm{s}) \mathrm{a}$ & 29 Aug. 98 & CCD & FP235 & 0.68 & $24 \times 180$ & 2.'0 & 2 "' 8 \\
\hline NGC & 615 & $\mathrm{SA}(\mathrm{rs}) \mathrm{b}$ & 29 Aug. 98 & $\mathrm{CCD}$ & FP235 & 0.68 & $24 \times 180$ & $2 . .5$ & 3.' 8 \\
\hline NGC & 972 & $\mathrm{SA}(\mathrm{rs}) \mathrm{b}$ & 21 Feb. 98 & $\mathrm{CCD}$ & FP235 & 0.68 & $24 \times 180$ & $2 . .6$ & 3.'5 \\
\hline NGC & 1084 & $\mathrm{SA}(\mathrm{s}) \mathrm{c}$ & 26 Oct. 95 & IPCS & FP501 & 0.92 & $32 \times 225$ & $2 . .0$ & 3". 5 \\
\hline NGC & 1134 & S..? & 23 Feb. 98 & $\mathrm{CCD}$ & FP235 & 0.68 & $24 \times 150$ & $2{ }^{\prime \prime} 4$ & $2 ! 9$ \\
\hline NGC & 2964 & $\mathrm{SAB}(\mathrm{r}) \mathrm{bc}$ & 01 Dec. 99 & $\mathrm{CCD}$ & FP235 & 0.68 & $24 \times 180$ & $2 . .0$ & $2 \prime .5$ \\
\hline NGC & 3583 & $\mathrm{SB}(\mathrm{s}) \mathrm{b}$ & 28 Feb. 00 & CCD & FP235 & 0.68 & $32 \times 180$ & $2{ }^{\prime \prime} 8$ & 3.' 5 \\
\hline NGC & 3893 & $\mathrm{SAB}(\mathrm{rs}) \mathrm{c}$ & 10 Dec. 96 & $\mathrm{CCD}$ & FP501 & 0.90 & $24 \times 180$ & $2 . .5$ & 3.' 1 \\
\hline NGC & 4100 & $\mathrm{R}^{\prime} \mathrm{SA}(\mathrm{rs}) \mathrm{bc}$ & 21 Mar. 96 & IPCS & FP235 & 0.77 & $24 \times 220$ & $2 . .5$ & 3.'5 \\
\hline NGC & 4136 & $\mathrm{SAB}(\mathrm{r}) \mathrm{c}$ & 21 Feb. 98 & $\mathrm{CCD}$ & FP235 & $1 " .36$ & $24 \times 180$ & $2 . .5$ & $4{ }^{\prime \prime} 0$ \\
\hline NGC & 4414 & $\mathrm{SA}(\mathrm{rs}) \mathrm{c}$ & 21 Feb. 98 & $\mathrm{CCD}$ & FP235 & $0 . ' 68$ & $24 \times 120$ & $2 . .0$ & 3.'0 \\
\hline NGC & 4814 & $\mathrm{SA}(\mathrm{s}) \mathrm{b}$ & 23 Feb. 98 & CCD & FP235 & 0.68 & $24 \times 120$ & $2 . .4$ & 3.'1 \\
\hline NGC & 5371 & $\mathrm{SAB}(\mathrm{rs}) \mathrm{bc}$ & 23 Mar. 96 & IPCS & FP235 & 0.77 & $24 \times 460$ & 4.'0 & 5.5 \\
\hline NGC & 6643 & $\mathrm{SA}(\mathrm{rs}) \mathrm{c}$ & 16 Мay 97 & $\mathrm{CCD}$ & FP235 & 0.45 & $24 \times 360$ & $1 . .5$ & $2{ }^{\prime \prime} 0$ \\
\hline
\end{tabular}

presence of several subsystems of ionized gas clouds on the line of sight. In these cases we have performed a multi-component Gauss analysis of such profiles to separate the kinematic components. The resulting errors in the "main component" velocity measurements are about $15-20 \mathrm{~km} \mathrm{~s}^{-1}$ for FP235 and $5-10 \mathrm{~km} \mathrm{~s}^{-1}$ for FP501 observations, which is similar to the errors of barycenter-based velocities in the case of a symmetrical line profile. To construct a velocity field in the regions with multicomponent profiles we use only those velocities which correspond to the "main' component". In practically all the cases its value appears to be nearest to the velocities found in the neighbouring regions with a one-component shape of an emission-line profile. With the exception of a complex situation in the central region of NGC 1084, such an approach has not created any velocity discontinuity. The examples of the emission line spectra in the regions with the "abnormal" profiles are plotted in Fig. 1. Double-peaked profiles may be found in the circumnuclear regions (NGC 23, NGC 1084, NGC 1134, NGC 2964, NGC 6643), as well as in the outer disks (IC 1525, NGC 1084, NGC 3893).

The most complex case is represented by the circumnuclear regions of galaxies with broad stellar $\mathrm{H} \alpha$ absorption lines in the spectra. Unfortunately, our spectral range is too narrow, and the IFP order overlapping often prevents a proper correction for this absorption feature. We met such situations in some regions in the centers of IC 1525, NGC 4136, NGC 4414, and NGC 5371. We have tried to use a double-Gaussian (emission + absorption) model to calculate the velocities in these regions. However the errors of the velocity measurements were too large $\left(\sim 50-70 \mathrm{~km} \mathrm{~s}^{-1}\right)$. Therefore we have excluded these regions from the resulting velocity fields (see the descriptions of the individual galaxies in Sect. 4).

\section{Results}

\section{1. "Kinematic" orientation parameters of galactic disks}

After gas velocity fields of galactic disks became available (first from HI radio line observations, later from optical line measurements), several ways were proposed to solve the problem of restoring rotation curves of galaxies from the obtained data. In most cases the basic assumption is to consider deviations of the observed velocities from those expected in a pure circular rotation picture as random ones, which have a "noisy" character:

$V^{\mathrm{obs}}=V^{\mathrm{mod}}+\Psi$

$V^{\mathrm{mod}}(r, \varphi) \equiv V_{\text {sys }}+V_{\text {rot }}(r) \cos \varphi \sin i$,

where $\Psi$ is a random deviation of the observed velocity from that predicted in the model; $V_{\text {sys }}$ is the systemic line-of-sight velocity of the galaxy; $V_{\text {rot }}$ is the model circular velocity of the gas in the plane of the galaxy which depends on the galactocentric radius $r ; \varphi$ is the galactocentric azimuthal angle measured with respect to the major axis; $i$ is the inclination (the angle between the galactic plane and the sky plane).

But even this very simplistic approach leaves the problem of determining the rotation curve to be rather complicated. In general, there are five parameters which should be found or taken from elsewhere in order to extract the rotation velocities from the line-of-sight velocity field. They are: the systemic velocity of a galaxy $V_{\text {sys }}$, the position of the center of mass $x_{0}, y_{0}$, the position angle of the line of nodes $\mathrm{PA}_{0}$ and the disk inclination angle to the line of sight $i$.

In early papers (Warner et al. 1973; Pence 1981) these parameters were assumed to be constant over the entire disk. In this case they could be found either from the analysis of photometric data (with the exception of $V_{\text {sys }}$ ) (Pence 1981), or by minimizing a sum of squared differences between the measured and model-predicted velocities for a pure rotation model (Warner et al. 1973; Pence 1981). In this case the rotation velocity at a given galactocentric radius was calculated as a weighted mean of all points lying in the plane of the disk within a ring of radius $r$ and of width $\mathrm{d} r$. Even in these early works it was revealed that different methods give discrepant results, which was ascribed to the influence of non-circular motion (Pence 1981). Note that a similar effect could also be caused by non-planar motion. As it was mentioned by van Moorsel \& Wells (1985), the Warner et al. (1973) method is of a trial-anderror nature, which makes it difficult to determine (cite) "what 

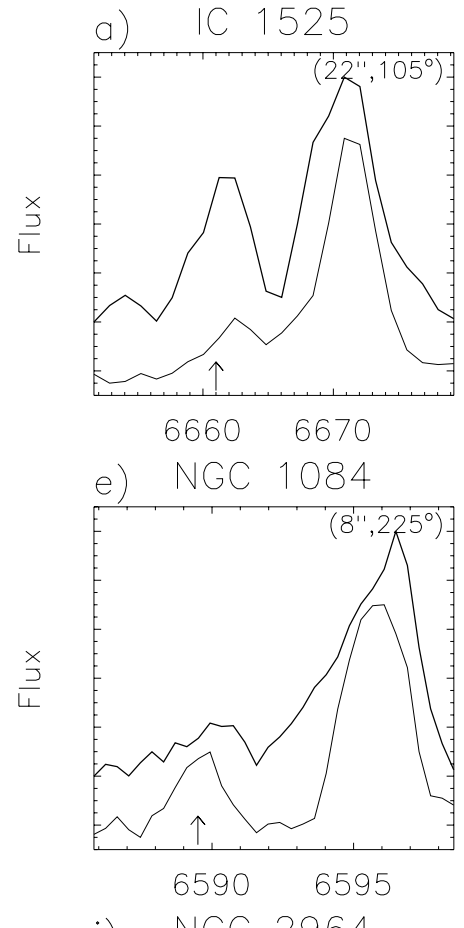

i) NGC 2964

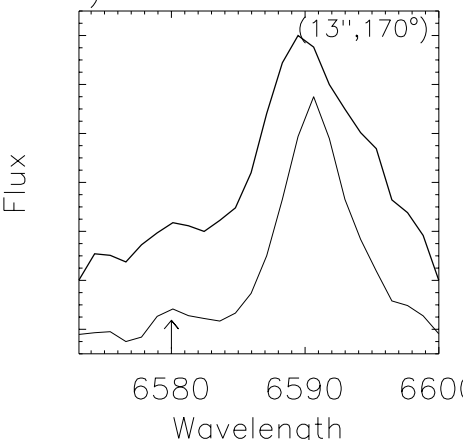

b) IC 1525

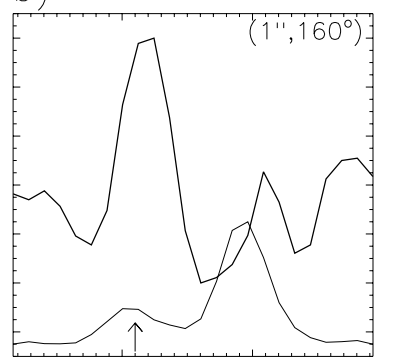

$6660 \quad 6670$

f) NGC 1084

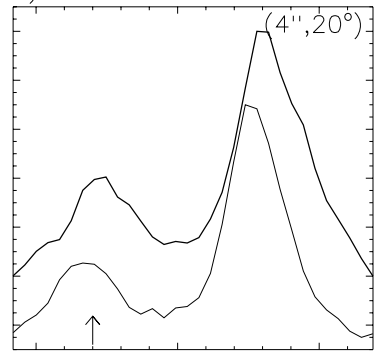

$6585 \quad 6590 \quad 6595$
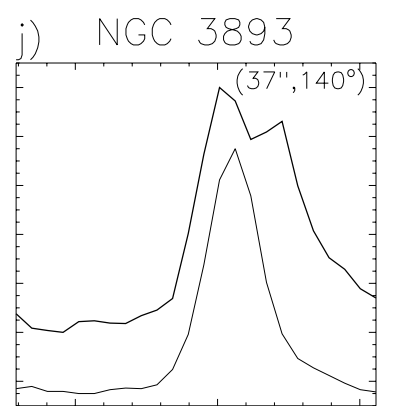

6580
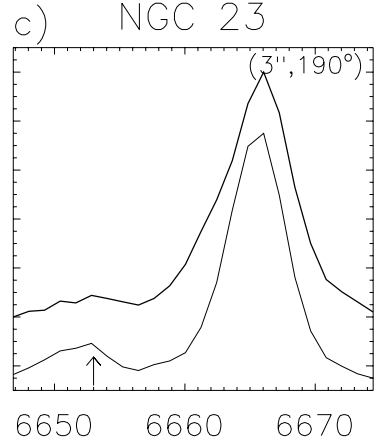

g) NGC 1134

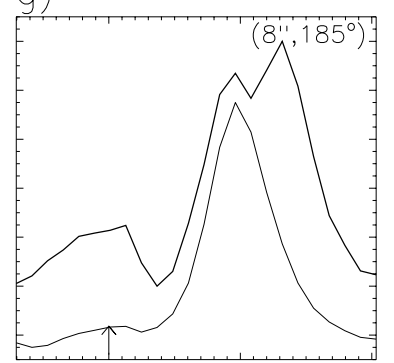

$66306640 \quad 6650$
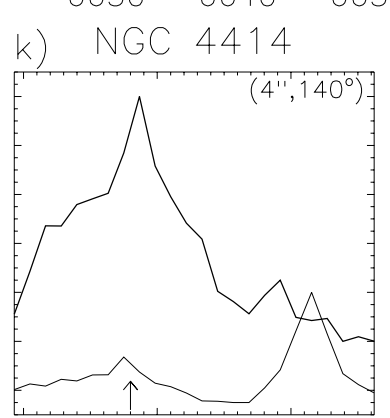

6586560
65706580

Wavelenath

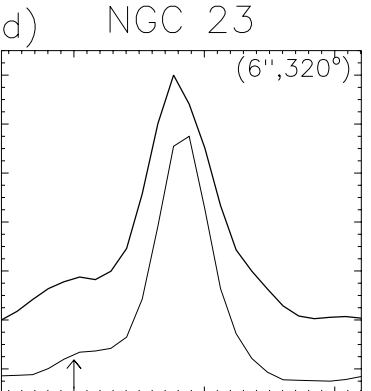

$6650 \quad 6660 \quad 6670$
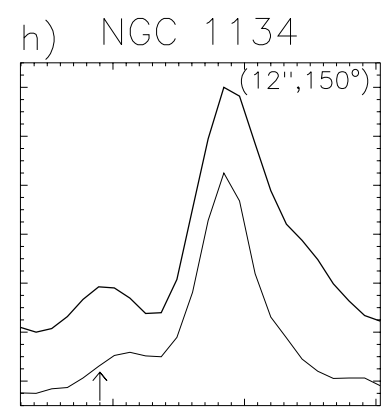

$66306640 \quad 6650$

l) NGC 6643

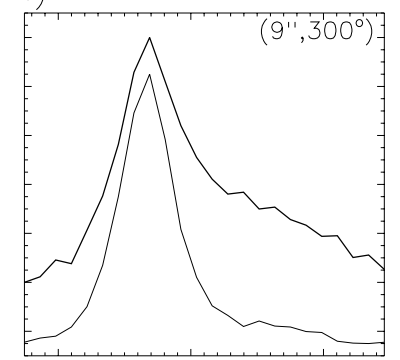

$65906600 \quad 6610$

Wavelenath

Fig. 1. The examples of the IFP-spectra with complex structure of the $\mathrm{H} \alpha$ emission-line profiles (see Sect. 4 for the detailed description of the individual galaxies). The thick line shows the spectrum extracted for a region with the cylindrical coordinates $(r, \mathrm{PA})$ given at the top-right corner of the each frame. These profiles reveal different kinds of peculiarities, such as blue/red wings (see c, d, e, f, h, i and l), double-humped structure (see g, j), H $\alpha$-absorption distortion (see b and k), and the [NII] emission line interference (see a, g, and h). The thin line reproduces the spectrum of the nearest galactic region with the "normal" (symmetrical) shape of the emission line profile. The arrows mark the position of the [NII] ( $\lambda 6548 \AA$ or $\lambda 6583 \AA$ ) line, intruding from the neighboring interference order. Note that the wavelength scale corresponds only to the $\mathrm{H} \alpha$ line velocities.

errors are to be assigned to the results". These authors proposed a modification of the ring method assuming a certain functional approximation of the rotation curve under the best-fitting orientation parameters; after that they solved the system of many equations for the total number of unknowns by a least-squares algorithm (without using any weighting based on the position inside of a galaxy). Unlike van Moorsel \& Wells, below we do not use any parameterization of the rotation curve, applying the least-square algorithm to the model of a thin circularly rotating disk where the velocity of rotation of a given ring is considered as the unknown.

The parameters $\mathrm{PA}_{0}$ and $i$ estimated in the early works were found in some cases to be inapplicable to the outermost parts of galaxies, where a distortion of isovelocities is often seen (see for example Warner et al. 1973, for M 33; or Rogstad et al. 1979, for NGC 300). To improve the procedure without rejecting a circular motion approximation, it was proposed (Begeman 1989) to consider the values of $\mathrm{PA}_{0}$ and $i$ (or in general, all the parameters involved) as functions of $r$. This approach is called "a tilted-rings model". In this model it is assumed that the disk of a galaxy may be described by a set of concentric rings with fixed values of $V_{\mathrm{sys}}, x_{0}, y_{0}, i$, and $\mathrm{PA}_{0}$ within each of them. In other words, each ring is allowed to have arbitrary circular velocity and orientation parameters. The values of $V_{\mathrm{sys}}, x_{0}$, and $y_{0}$ are often taken as the same for all the rings. This approach is widely used to determine rotation curves ${ }^{2}$.

2 Note however that in some papers (see Schommer et al. 1993; Amram et al. 1995; Garrido et al. 2002, 2003) the authors use constant values of orientation angles $\mathrm{PA}_{0}$ and $i$ for each galaxy as the best fit parameters, which may be found in the frame of circular rotation model by the least-square method or by iterations. 


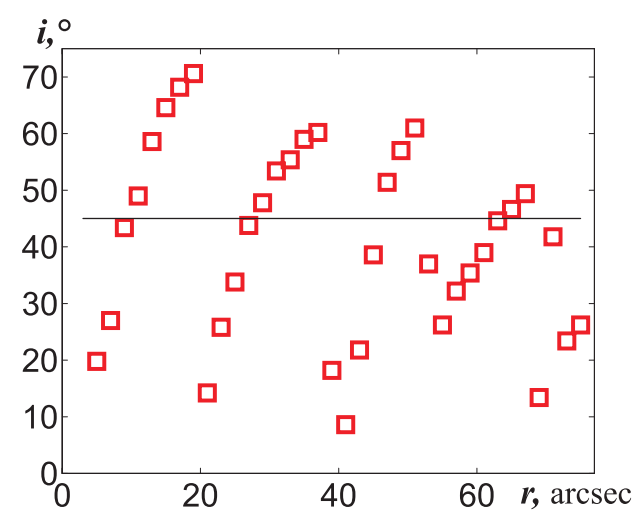

(a)

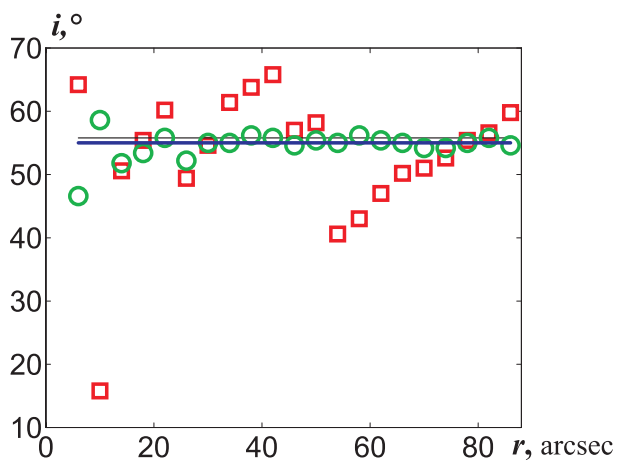

(c)

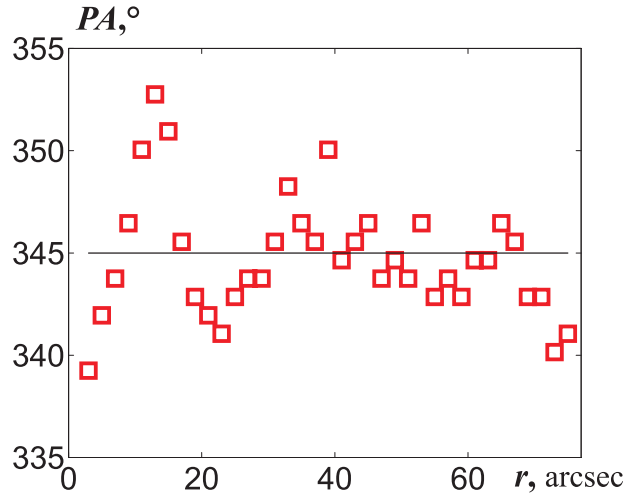

(b)

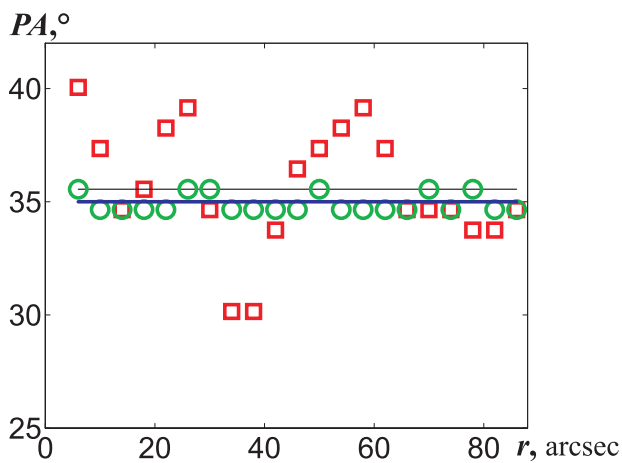

(d)

Fig. 2. Radial variations of the inclination angle (left) and position angle of the line-of-nodes (right) found by the tilted-rings method applied to the observed velocity field of NGC 3893 (upper diagrams) and to the artificial galaxy (below), in which the velocity field is a sum of pure rotation, non-circular regular velocities expected in the simplified model of density waves, and "noisy" velocity dispersion of about $10 \mathrm{~km} \mathrm{~s}^{-1}$. Thin lines in the lower diagrams mark the values of parameters obtained under the assumption of unique orientation parameters for the whole velocity field. Circles are the estimates made for the model in absence of density waves. Thick lines are real values of parameters accepted in the model.

Variations of the parameters are allowed not only at the periphery of a disk, that may be physically advocated because of a possible warp, but at all radii. It is worth mentioning that although the warping of gaseous (and sometimes stellar) disks of galaxies is a well-known fact, significant variations of inclination usually take place in the outermost parts of galaxies and, as a rule, beyond the regions covered by optical spectral measurements. It seems that the parameter variations obtained by formal use of the tilted-rings model is often a consequence of non-circular motions which are not taken into account by this method. If the variations of either $i$ or $\mathrm{PA}_{0}$ (or $x_{0}, y_{0}$ ) bear a systematic character, it may be an indication that the presence of a spiral density wave (or bar) should be taken into account (Lyakhovich et al. 1997; Fridman et al. 1997).

As an illustration, Figs. 6 and 2a,b show the radial variations of $i$ and $\mathrm{PA}_{0}$, formally determined by the tilted-rings method for NGC 3893 - one of the galaxies of our sample with rather moderate inclination. The systemic velocity and center position were assumed to be invariable.

In Figs. 2c, d analogous dependencies are shown for an artificial (model) velocity field. This field was created as a sum of pure rotation, non-circular regular velocities expected in the simplified model of two-armed density waves, and a "noisy" non-correlated component imitating the errors of the velocity measurements and the occasional small-scale velocity dispersion. For definiteness, the amplitude of the rotational component was taken to be $210 \mathrm{~km} \mathrm{~s}^{-1}$, and of the regular non-circular velocities $-10-20 \mathrm{~km} \mathrm{~s}^{-1}$, and the root mean square deviation of the "noisy" component was taken as $10 \mathrm{~km} \mathrm{~s}^{-1}$. Circles mark estimates of the parameters when regular non-circular velocities induced by the density wave are excluded. One can see that dependencies obtained for the model which includes the density waves reproduces the radial variations of parameters formally found for the real galaxy rather well. It is also clear that when the density wave is eliminated from the model velocity field, the random motions may lead only to small parameter variations (by 1-2 degrees). It seems that the large variations of parameters in the tilted-rings model (Figs. 2a,b) may be naturally explained by the presence of a density wave.

The natural starting point for determining the orientation parameters is an assumption that they remain constant over all the optical part of a disk. But this in itself is not enough to find the angles we seek in a correct way if regular velocity perturbations in density waves take place. The main problem is that the latter introduce systematic, non-occasional deviations from the pure circular motion, that do not allow the use of the 
representation of the measured line-of-sight velocity in the form (1). Due to the same reason, the orientation parameters calculated for a given ring may be shifted in a systematic, non-occasional way from their real values. It also renders it useless to seek the most probable values by averaging the data obtained for different rings, as it takes place often (see for example Carignan et al. 1988; Begeman 1989; Beauvais \& Bothun 1999, 2000, 2001).

In order to not resort to the complex and laborious method of constructing a self-consistent model of non-circular motion in the disk, which being applied to an observational velocity field may give the best fit parameters (see for example Fridman et al. 2001a,b), one may use a simplified approach. The idea was proposed by Fridman et al. (2001a), where a radial periodicity of motion in the density wave leading to a periodical character of the wave contribution into the Doppler velocity component is suggested. Hence, if one is to seek the best values of the orientation parameters, one can expect that among the methods ignoring the density wave component of non-circular motion, the best method uses the velocity field as a whole, without estimating individual parameters for separate rings. In this case the contributions of systematic deviations from circular rotation in different parts of the disk are self-compensated, at least partially.

Actually this approach (without discussing the role of noncircular motion) was first used by Warner et al. (1973). Our basic principles of the determination of rotation curves remain the same as those used by Warner et al. (1973) aside from the different approach to weighting the data.

A consistent and reliable way to estimate different components of the Fourier spectrum of the line-of-sight velocity field (and particularly the rotation velocity) was proposed and validated by Lyakhovich et al. (1997), Fridman et al. (1997, 2001a), Burlak et al. (2000). It is based on the application of a least-square method to the expansion of the azimuthal distribution of the observed gas velocities into the truncated Fourier series. The rotational velocity is allowed to vary from one ring to another. This is the only parameter which runs along the radius in our model.

For a given ring of radius $r_{1}$ and width $\mathrm{d} r$ a sum of squared deviations of the measured velocities from those expected in the model of pure circular rotation is:

$\chi^{2}\left(r_{1}\right)=\sum_{j}^{N_{\mathrm{l}}}\left(V_{j}\left(r_{j}, \varphi_{j}\right)-V_{\text {sys }}-V_{\text {rot }}\left(r_{1}\right) \cos \varphi_{j} \sin i\right)^{2}$.

Here the summation is performed over all $N_{1}$ points of the ring where the line-of-sight velocity was estimated, and the weighting coefficients are assumed to be equal ${ }^{3}$. Analogous

\footnotetext{
${ }^{3}$ We ignore a weighting function in this work. The use of $\cos \varphi$ as a weighting function is hardly justified here because the parameters we are seeking are determined not from combining individual velocity measurements, but rather from the functional dependence of the lineof-sight velocities on the azimuthal angle, the expression for which is supposed to be known. In this case each point has a weight which does not depend on $\varphi$. See also Begeman (1989) who wrote (cite): "... In principle there is no objection to the use of equal weights, except when the uncertainties in the determination of the radial velocities are
}

expressions may be easily written for any choice of the weighting function. For given values of the center position, systemic velocity, inclination, and position angle of the line of nodes the minimum of $\chi^{2}\left(r_{1}\right)$ is reached under $\partial \chi^{2}\left(r_{1}\right) / \partial V_{\text {rot }}\left(r_{1}\right)=0$, that gives:

$V^{\mathrm{rot}}\left(r_{1}\right)=\frac{1}{\sin i} \frac{\sum_{j} \cos \varphi_{j}\left(V_{j}\left(r_{j}, \varphi_{j}\right)-V_{\mathrm{sys}}\right)}{\sum_{j} \cos ^{2} \varphi_{j}}$.

For complete filling of the ring by the measurements, this expression is reduced to a familiar one for the reverse Fourier transformation.

Under condition (3) the quadratic sum of deviations in a ring (2) takes the form:

$$
\begin{aligned}
\chi^{2}\left(r_{1}\right)= & \sum_{j}\left(V_{j}\left(r_{j}, \varphi_{j}\right)-V_{\mathrm{sys}}\right)^{2} \\
& -\frac{\left[\sum_{j} \cos \varphi_{j}\left(V_{j}\left(r_{j}, \varphi_{j}\right)-V_{\mathrm{sys}}\right)\right]^{2}}{\sum_{j} \cos ^{2} \varphi_{j}} .
\end{aligned}
$$

For the whole disk we have:

$\chi^{2}\left(x_{0}, y_{0}, i, \mathrm{PA}, V_{\text {sys }}\right)=\sum_{l} \chi^{2}\left(r_{1}\right)$

Some parameters contained in the brackets on the left side of Eq. (5), (say, the center position and/or systemic velocity) may be considered as knowns. The others may be found by minimizing the expression (5). In practice we estimated $V_{\text {sys }}$ and the center position as the best fit symmetry center of the observed velocity field by comparing the velocities of points situated symmetrically with respect to the center, and kept these values fixed.

If the rotation curve has a steep gradient in the central part of a galaxy, it may be more convenient to use a slightly different version of the expression for a quadratic sum of deviations. It may be obtained in a similar way as written above, but starting from a constant angular velocity for a given ring instead of a constant linear velocity. The resulting expression differs from Eq. (4) by the replacement of all values of $\cos \varphi_{j}$ onto $x_{j}=r_{j} \cos \varphi_{j}$.

For the velocity field of the model galaxy which includes density wave perturbations, the parameters $i$ and $\mathrm{PA}_{0}$ found by the method described above are shown by thin lines in Figs. 2c and $\mathrm{d}$. They coincide with the parameters of the model (thick lines) within a range of under one degree. It enables us to conclude that the method gives correct estimates of the orientation parameters, even in the presence of systematic non-circular motions in a galaxy.

known to be different. (In general these uncertainties are not known.)" However he adds: "The use of non-uniform [azimuthal] weights [is] preferable... radial velocities in positions close to the minor axis carry less information about the underlying circular velocity than at positions close to the major axis. Also by this reason one should give more weight to positions close to the major axis". Indeed the latter statement is correct only when the circular rotation is restored from the individual local velocity estimates, not from the comparison with the model dependencies expected for a flat disc. 
Table 3. Orientation parameters of galaxies determined by the different methods.

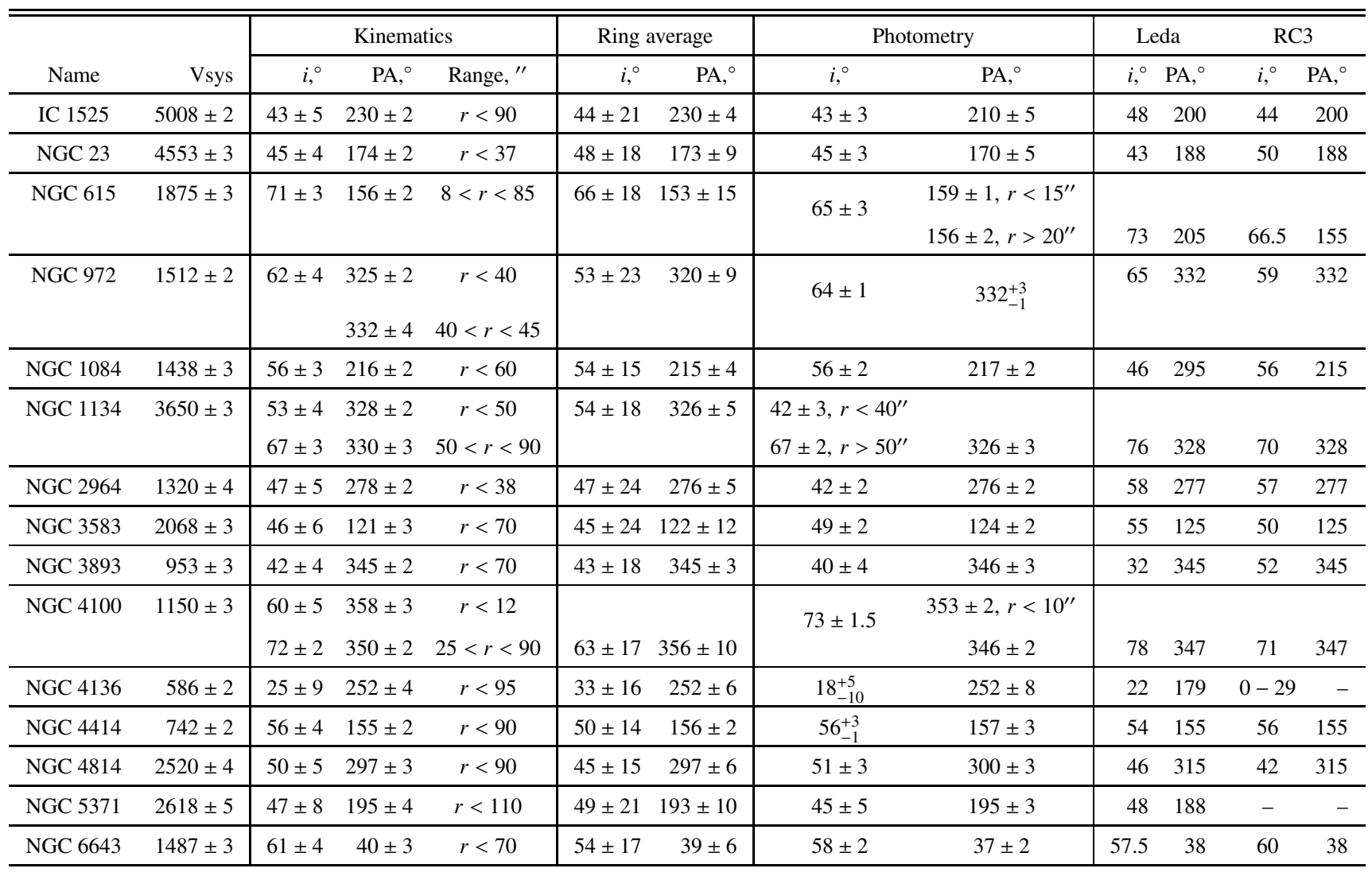

"Kinematic" orientation parameters of our sample galaxies, obtained by this method, are presented in the third column of Table 3 . Their calculation was made by minimizing the expression (5) with fixed positions and systemic velocities of the center. Confidence intervals given in Table 3 were estimated from the formally determined errors of the mean values in a model of circular rotation and from the shape and width of the profile of $\chi^{2}\left(i, \mathrm{PA}_{0}\right)$ near its minimum. The obtained results are insensitive to the chosen width of rings until it is smaller than the radial scale of velocity variations in a galaxy. The $\mathrm{PA}_{0}$ is traditionally measured counter-clockwise from the North direction to the receding part of the major kinematic axis of the galaxy.

Note that our method of interferometer data calibration may introduce some small systematic errors in systemic velocity estimation (however these errors do not influence the measurements of relative velocity variations in the line-of-sight velocity field). A comparison of our $V_{\text {sys }}$ (the second column in Table 3) with the NED database values shows that the velocity difference is about $10-50 \mathrm{~km} \mathrm{~s}^{-1}$ for different objects - that is of the same order as the scatter of estimates obtained by different authors.

The fourth column of Table 3 contains the kinematially determined orientation parameters obtained by a formal averaging of values found for different rings (a classical tilted-rings model). Because of the non-random variations of the orientation angles of individual rings, it is incorrect to characterize the confidence intervals of these estimates by the formal value of the error in mean. For this reason, a root mean square deviation from the mean value was taken as a confidence interval. As expected, this method of averaging gives estimates that are not as reliable as those in the method we have preferred above.

\section{2. "Photometric" orientation parameters of galactic disks}

Photometric methods are widely used because they are much simpler and more understandable and do not demand analysis of the detailed velocity fields. The methods are usually based on estimating ellipticity and isophote orientation by fitting ellipses to the outermost isophotes which can be reliably traced (they are assumed to be undisturbed by disk warping or by internal absorption). Another, more sophisticated way of getting the results is to use azimuthal Fourier analysis of brightness distribution of a galaxy in the plane of sky (Grosbol 1985) or in the plane of the galactic disk (Iye et al. 1982).

As in the case of kinematic methods, the main problem of the photometric approach is to take into account the deviations from the axial symmetry caused by spiral arms or a bar. It may be especially severe when a galaxy does not possess a radial zone wide enough where the brightness distribution stays axisymmetric. Ignoring this problem may lead to large systematic errors in evaluating the orientation parameters.

In a formal sense, the second Fourier harmonic may appear as a result of erroneous orientation parameters which are used for the deprojection of an axially symmetrical disk. This harmonic also appears if the parameters used are correct, but 
non-symmetrical details such as spirals or a bar are present, so it is impossible to separate these factors within the frame of Fourier analysis.

The only way to solve the problem is to use differences in the phase and amplitude behavior of the second Fourier harmonic when different factors are responsible for its appearance. Particularly, the amplitude of the second harmonic caused by an error in the estimation of the inclination grows as this error increases. If the inclination is underestimated, the line of maxima of the second harmonic would align with the major axis because of this error, and in the opposite case it would coincide with the minor axis. Unlike this behavior, the amplitude of the second harmonic produced by a density wave should weakly correlate with the orientation of the disk. Hence, to carry out a Fourier analysis of the azimuthal brightness distribution under initial assumption of zero inclination, and to draw the line of maxima of the second harmonic, one can get the orientation of the disk major axis (see also Grosbol 1985, who used a similar approach for the outer parts of real galaxies). The most clearly delineated orientation of the line of maxima along the major axis would be observed in the regions of a weak density wave (that is for a poorly traced spiral structure or a bar), and even if the width of each such region is rather small, coincident directions of the lines of the harmonic maxima over all these regions enable us to estimate an orientation of the major axis $\mathrm{PA}_{0}$ with good accuracy. It is essential that the method does not demand these free-of-distortion regions to be preliminarily found in a galaxy: they reveal themselves in the process of the second harmonic phase calculations.

The inclination angle $i$ may be found (in the absence of the non-axisymmetrical structure) as the angle where the phase of maxima of the second harmonic of the deprojected image switches from the major axis to the minor one. In the presence of a density wave the interference of two factors (the error of $i$ and the influence of the spiral structure) causes the phase of maxima of the second harmonic to be shifted into the position between the major (or minor) axis and the observed brightness maximum of a spiral arm in the given annulus. In the annuli where the spiral arms have the lowest contrast (they may be revealed during the procedure of $\mathrm{PA}_{0}$ determination) the phase shift should be the least. Let us note that when the probed inclination passes through its correct value, the line of phase of the second harmonic turns shortly in these regions because of the jump (at $90^{\circ}$ ) of the position of the maximum contribution of the error of $i$ in this harmonic. It allows us to estimate $i$ as an angle where the phase of the second harmonic best fits the observed spiral pattern (including the regions of low brightness of the arms).

The photometric method described above has been successfully applied to a sample of double-barred galaxies by Moiseev et al. (2004); the reader may find some details of practical use of this method in the cited paper. The results of "photometric" estimation of the orientation parameters for the present sample galaxies are given in the fifth column of Table 3 . The confidence intervals characterize an uncertainty caused by the nonvanishing amplitude of the density wave. For the imaging data we used (red) continuum brightness distributions obtained during our observations with IFP. For three galaxies the published
I-band images obtained at the $1 \mathrm{~m}$ "Zeiss-1000" telescope of the SAO RAS were used. These are NGC 615 (Sil'chenko et al. 2001), NGC 1084 (Moiseev 2000), and NGC 1134 (Bizyaev et al. 2000). The $R$-band image of NGC 4136 obtained at the $1.1 \mathrm{~m}$ telescope of the Lowell Observatory was taken from the Digital Atlas of Nearby Galaxies (Frei et al. 1996). For some galaxies raw photometric data from the ING Archive of the UK Astronomy Data Centre were also retrieved. These are the observations of NGC 23 in $R$-band and NGC 6643 in $I$-band at the $1 \mathrm{~m}$ Jacobus Kapteyn Telescope and the observations of NGC 4100 and NGC 6643 in $R$-band with the $2.5 \mathrm{~m}$ Isaac Newton Telescope. The K-image of NGC 972 was obtained with the UKIRT telescope (Hawaii) and is kindly provided by Dr. S. D. Ryder.

\subsection{A comparison of "kinematic" and "photometric" estimates of orientation parameters}

It is convenient to compare the estimates of $i$ and $\mathrm{PA}_{0}$ presented in Table 3 as follows: 1 ) by comparing our "kinematic" parameters obtained for the whole velocity field with those taken as the average of the parameters found for individual rings in a tiltedrings model; 2) by comparing our "photometric" parameters with the inclination and position angles contained in the LEDA and RC3 Catalogue; and 3) by comparing the "kinematic" estimates with the "photometric" ones.

\subsubsection{The whole field versus ring average method}

The confident intervals for the parameters $i$ and $\mathrm{PA}_{0}$ obtained as the means over individual rings are much larger than those from our kinematic estimations. Although our whole-field estimates formally fall into the wide confidence intervals of the "tilted-rings model" results, so that both could be considered as "consistent" the whole velocity field is significantly higher than that using the average of parameters found for individual rings in a tiltedrings model $^{5}$.

\subsubsection{Photometric method taking into account spiral structure versus outer-isophote methods}

An agreement between our estimates of $i$ and $\mathrm{PA}_{0}$ obtained by the photometric method taking into account the regular spiral structure with those listed in the LEDA database and RC3 catalogue appears to be rather moderate. Note however that the accuracy of the LEDA and RC3 data is certainly lower than ours as they are based on small-scale Palomar images of galaxies. Concerning LEDA, one of the parameters $i$ and $\mathrm{PA}_{0}$ are "consistent" (within $\sqrt{2} \Delta_{1}$ ) for about a half of the objects, and only for 3 galaxies (NGC 972, 4414, and 6643) the

\footnotetext{
4 Formally speaking, two values $a \pm \Delta_{1}$ and $b \pm \Delta_{2}$ are "consistent" if $|a-b| \leq \sqrt{\Delta_{1} 2+\Delta_{2} 2}$.

5 We note above that it is not correct to use the statistical error of the means as the measure of the confidence interval when averaging over individual rings. However, if one does it formally, the difference between the angles estimated for the whole field and by averaging over rings would be greater than the confidence interval.
} 
"consistency" exists for both orientation parameters. A comparison with the RC3 catalogue gives better results: inclination estimates are "consistent" for 8 of 14 and $\mathrm{PA}_{0}$ - for 10 of 13 common galaxies. Both parameters are "consistent" simultaneously for 6 galaxies.

Nine galaxies of our sample are also included in the list of objects for which Grosbol (1985) estimated the orientation parameters by analyzing the second Fourier harmonic of the azimuthal brightness variations on small-scale Palomar images at several radial bins (IC 1525, NGC 23, 2964, 3583, 3893, $4136,4414,4814,5371)$. The root mean square difference between his values and ours obtained by the photometric method is $6^{\circ}$ for $i$ and $11^{\circ}$ for $\mathrm{PA}_{0}$. The root mean square differences between Grosbol's and LEDA data are slightly higher, $8^{\circ}$ for $i$ and $12^{\circ}$ for $\mathrm{PA}_{0}$ (even if to exclude NGC 4136 with strongly discrepant estimates of $\mathrm{PA}_{0}$ ).

\subsubsection{Kinematic versus photometric parameters}

A comparison of the estimates of $\mathrm{PA}_{0}$ we obtained by the kinematic and photometric methods described in the previous subsections reveals discordant results in 3 of 15 galaxies (IC 1525, NGC 972, and NGC 4100). For the first galaxy the disagreement is most likely due to the presence of a rather strong bar. For NGC 972 the discordant estimates may also be related to the bar. Although this galaxy is commonly classified as a non-barred one, the presence of the inner bar is found in the infrared (see the next section). In NGC 4100 the difference of $\mathrm{PA}_{0}$ found by two methods is rather low (if to exclude the inner part, the kinematic and photometric $\mathrm{PA}_{0} \mathrm{~s}$ are $350 \pm 2$ and $346 \pm 2$ degrees respectively) and may be coincidental.

The non-circular motion caused by spiral density waves can distort our kinematic estimates of $\mathrm{PA}_{0}$, but these distortions are different in different parts of the disk and as a result they partly compensate each other when the whole velocity field is taken for the $\mathrm{PA}_{0}$ determination. The presence of a bar may produce stronger non-circular motion with the preferred orientation that are hard to compensate by averaging. It may explain the differences between the orientation parameter estimates obtained in some barred galaxies by the two methods. Figures 3 and 4 demonstrate the shapes of the lines of maxima of the second Fourier harmonics of the surface brightness for the barred galaxies IC 1525 and NGC 972 calculated using different estimates of $\mathrm{PA}_{0}$. As one may see, the shape of the lines calculated with the $\mathrm{PA}_{0}$ kinematically estimated (left) disagrees with the observed spiral arms. Instead, these lines tend to oscillate around some preferred direction which is close to the orientation of the major axis according to the photometric estimate of $\mathrm{PA}_{0}$. The shapes of the lines calculated with the latter $\mathrm{PA}_{0}$ values (right) are in accordance with the spiral arms traced by eye. All these considerations allow us to suggest that the true orientation parameters of these two barred galaxies should be closer to those derived from the photometric data.

Concerning the inclination $i$, its values found by us from kinematic and photometric data are "consistent" in 13 galaxies of 15. Those two galaxies (NGC 615 and NGC 1134) where the agreement is absent demonstrate rather complex asymmetric structure.

Indeed, we would advise use of both methods together when trying to estimate galaxy orientation parameters: a convincing reason is that the non-axisymmetry of a galactic disk a bar or a strong density wave - affects the parameter estimations by photometric and kinematic methods in a different way.

\subsection{Rotation curves in a model of pure circular gas motion}

Figure 5 shows the results of data processing for the galaxy IC 1525. Similar illustrations for other galaxies of our sample are presented in the electronic version of the article. For each galaxy the first three plots show the images in the red continuum and $\mathrm{H} \alpha$ (gray-scaled in proportion to the square root of the intensity), and line-of-sight velocity field of ionized gas with the isovelocity lines superimposed with a step of $50 \mathrm{~km} \mathrm{~s}^{-1}$ (the thick line corresponds to the systemic velocity $V_{\text {sys }}$ ). The coordinates of the center of a galaxy and its systemic velocity were found from kinematic data as the point of the best mirror symmetry of the velocity fields for all pairs of pixels taken on opposite sides with respect to the center. A small variation of the center coordinates has little effect on the orientation parameter estimates because they are influenced by different Fourier harmonics of the velocity field (see Lyakhovich et al. 1997). Additionally, we have determined the positions of the center from both photometric and kinematic data independently for several galaxies to be sure that they coincide within 1-2 pixels and it is always the case.

In the last plot the rotation curve is shown (the upper curve). It was calculated from the observed velocity field (see Eq. (3)) as the best fit curve of circular rotation for the "kinematic" orientation angles $i$ and $\mathrm{PA}_{0}$, which were assumed to be constant along the radius. The lower curves are the variations of $i(r)$ and $\mathrm{PA}_{0}(r)$ obtained for the individual rings in a tilted-rings model.

Since each elliptical ring contains from several tens to several hundreds of measured points, formal errors of the mean $V_{\text {rot }}(r)$ in the most cases are lower than $1-5 \mathrm{~km} \mathrm{~s}^{-1}$. However, as it was pointed out by Lyakhovich et al. (1997) and Fridman et al. (1997), the rotation curve obtained in the frame of the model of pure circular motion may have a systematic error of the order of non-circular velocities in a density wave. For this reason, with the error bars we mark the mean residual velocity in the individual rings $\left(\sqrt{\chi^{2}\left(r_{1}\right) / N_{1}}\right.$, see Eq. (4)). This way is evidently more correct than using formally determined errors of $V_{\text {rot }}(r)$ because it demonstrates a real confidence in these estimates. Genuine circular rotation curves, which may be found by methods taking into account the regular non-circular motion, will be within the error bars of the curves presented here. The large error bars seen at some rotation curves give evidence for significant deviations from the circular motion model rather than for the low accuracy of measurements. 


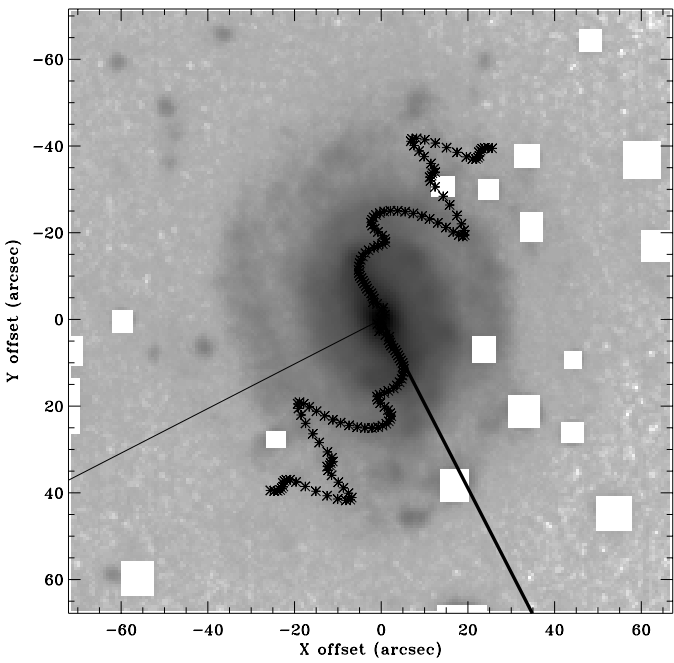

(a)

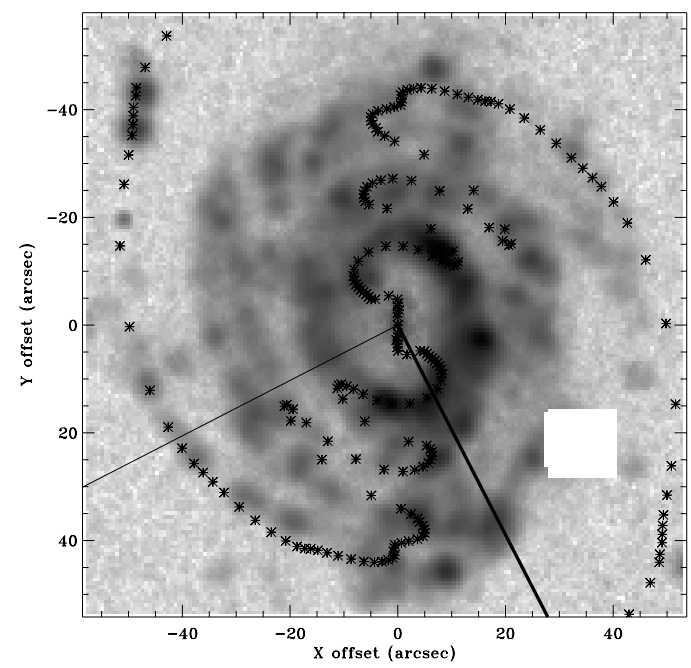

(c)

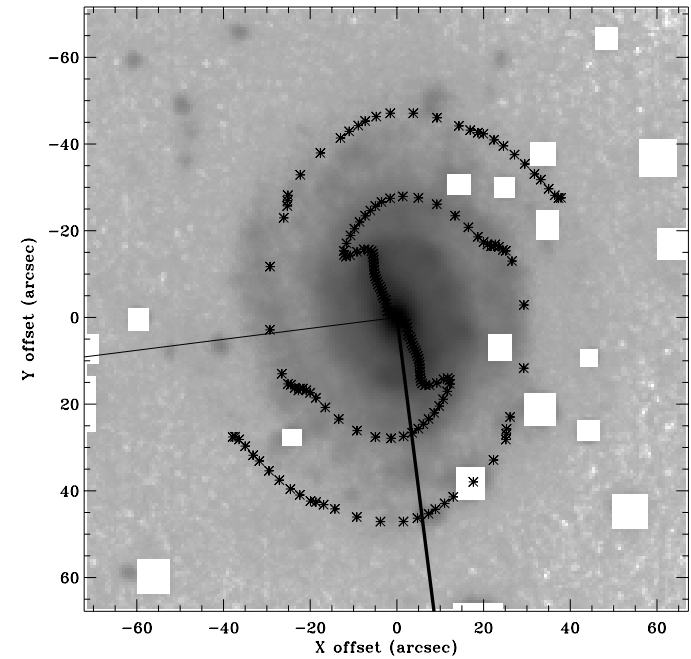

(b)

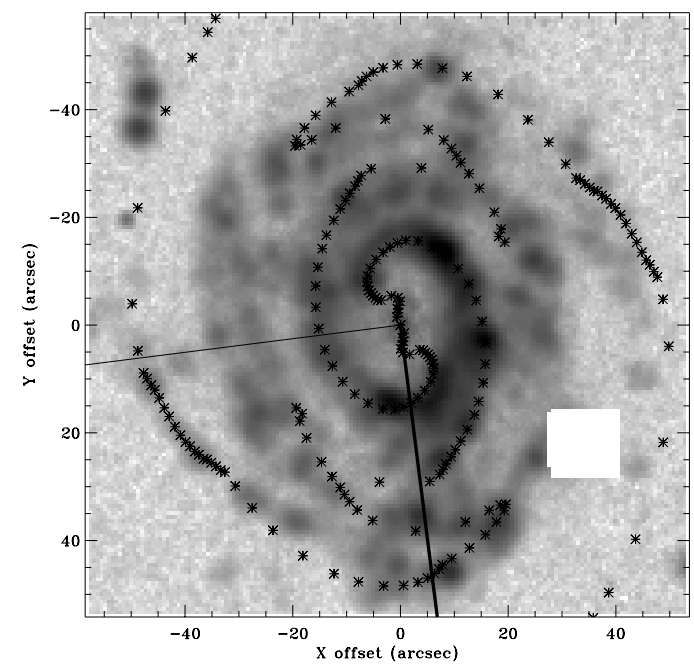

(d)

Fig. 3. A position of maxima of the second Fourier harmonic of the surface brightness calculated for different choice of $\mathrm{PA}_{0}$ superimposed onto the image of IC 1525. Left (a), c)): $\mathrm{PA}_{0}$ is chosen according to the kinematic data analysis. Right (b), d)): this parameter was taken from the photometry analysis (see the text). Images are in the red continuum (above) and in $\mathrm{H} \alpha$ line (below).

\section{Discussion}

Our sample of galaxies is rather widely representative, because it includes objects with a variety of kinematic properties of gas and with a wide range of structural features. The rotation curves in all the cases rise steeply in the inner parts within $r \approx 10^{\prime \prime}-20^{\prime \prime}$ (in most cases inside $1-3 \mathrm{kpc}$ from the center) due to the combined potentials of the bulge and the disk; further from the center the shape of curves looks different: the curves have either a plateau with some local velocity variations, or pass local maxima (as in the cases of NGC 23, 615, 1134, 3583, 5371). The curves of rotation in IC 1525, NGC 972, 1084, $3893,4100,4136,6643$ rise to the limit of the observed part of the galaxies (which, being less than the optical sizes of the galaxies, nevertheless contains the brightest part of the spiral structure). The opposite tendency is observed in NGC 2964; its rotation curve falls beyond the region of bright spiral arms.
The observed large-scale non-circular motions are usually connected with a spiral density wave or a bar. In particular, these motions result in the artificial $\mathrm{PA}_{0}$ and $i$ radial variations in a tilted-rings model, even when their real values remain constant. Systematic perturbations of the line-of-sight velocities in the region of spiral arms are most clearly seen in NGC 23, 4814, and 6643 (see, respectively, Figs. 6d, 17d and 19d), although in other galaxies they also appear.

In the presence of a bar (which can be considered another manifestation of a density wave) or some other type of triaxial structure (triaxial bulge, oval lense, etc) gas clouds follow elongated orbits which results in an observed turn of the kinematic major axis and leads to a well-known S-shaped distortion of isovelocities (see e.g. Chevalier \& Furenlid 1978). Besides that, in such cases a secondary component of the emission line is often observed; as a rule, it traces radial gas flows along the bar. Indeed, these peculiarities are observed in our 


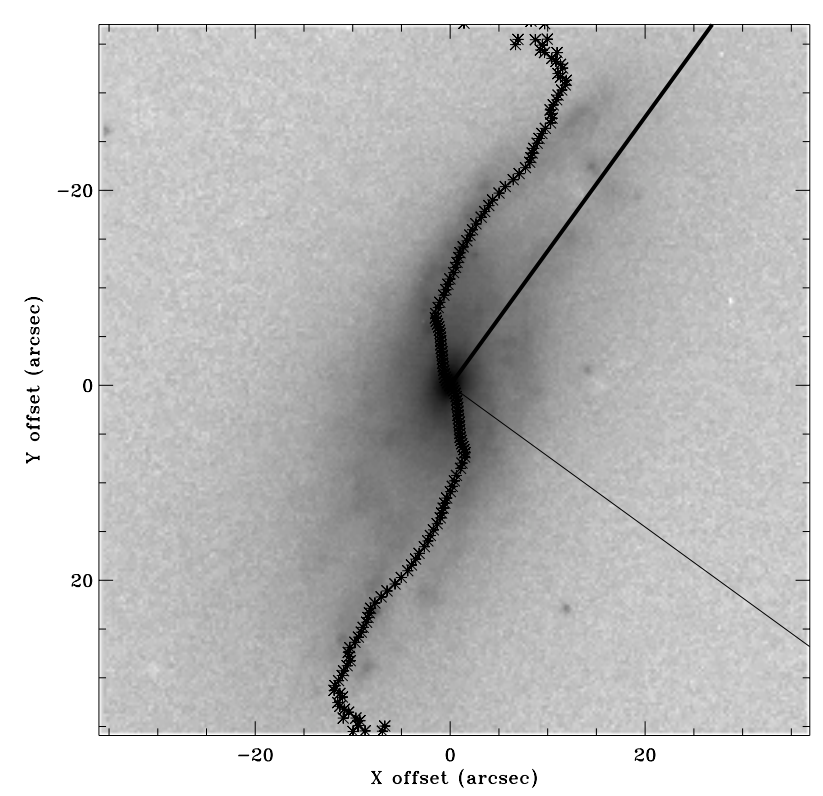

(a)

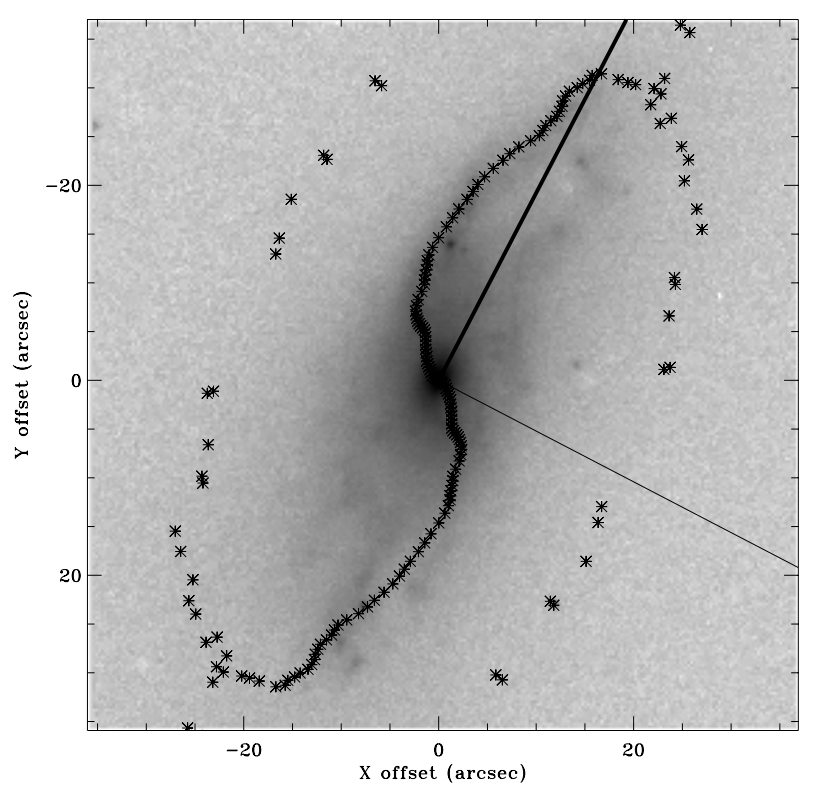

(b)

Fig. 4. A position of maxima of the second Fourier harmonic of the surface brightness calculated for a different choice of $\mathrm{PA}_{0}$ superimposed onto the $K$-band image of NGC 972. Left (a)): $\mathrm{PA}_{0}$ is chosen according to the kinematic data analysis. Right (b)): this parameter was taken from the photometry analysis.

galaxies, but curiously, the velocity anomalies correlate weakly with the relative size and contrast of a bar. There are three galaxies in our sample classified as SB in both NED and LEDA catalogs: IC 1525, NGC 23, and NGC 3583. In the former two we observe only a slight hint of bar-related anomalies, and only in NGC 3583 the expected distortion is clearly observed. In general, among the galaxies with the kinematic signatures of a bar there are the galaxies with the known photometric bars (SAB and SB, according to NED: IC 1525, NGC 23, NGC 2964, NGC 3583) as well as the galaxies previously classified as SA (NGC 615, NGC 972, NGC 4414, NGC 4814). However it is well known that low-contrast optical, or only infrared, triaxial structures may exist in galaxies classified as SA (NGC 972, NGC 615). Indeed, in NGC 972 the bar has been detected from the NIR surface photometry (Mayya et al. 1998), and in NGC 615 an oval inner disk has been found from the optical photometric data (Sil'chenko et al. 2001). We think also that the local regions with large residual gas velocities, situated symmetrically on opposite sides of the center and characterized by an enhanced [NII] emission, which are found in NGC 1084 and NGC 1134, may be connected to the front of a bar-induced shock wave, although optical bars are not clearly seen here.

Besides the large-scale deviations from circular rotation related to a bar or spiral arms, significant small-scale velocity anomalies are also observed in all galaxies we studied. They may be divided into three categories.

The first type refers to the non-circular motions of different natures in circumnuclear regions (within ten arcseconds from the center). Wide wings or significant shifts of spectral lines may be evidence for radial gas motion as a result of nuclear activity or an active star formation in the nuclei (IC 1525, NGC 23, NGC 972, NGC 1084). One should bear in mind, however, that similar manifestations (especially from observations with a moderate spatial resolution) may be caused by nuclear mini-bars (NGC 615, NGC 972, NGC 2964, NGC 4414) or by an inclined nuclear disk (NGC 4100). More detailed analysis is needed to distinguish between these cases (see discussion in Zasov \& Moiseev 1999).

The second type of local non-circular motion is observed in the bright HII regions of a disk. Among our galaxies such motions are definitely observed only in the most actively starforming galaxy NGC 972 . The velocity distortions are not too high (about $20 \mathrm{~km} \mathrm{~s}^{-1}$ ). This type of motion is most certainly caused by gas outflow as a result of heating and blowing by massive stars in the region of a local star formation burst. More energetic motions of this type are usually observed in starforming dwarf galaxies (radial expansion with amplitudes of ten to a hundred $\mathrm{km} \mathrm{s}^{-1}$, see for example Pustilnik et al. 2001).

The third type of local non-circular motion is the most enigmatic. It takes place far from the nuclei, in the regions with a typical diameter of about $0.3-1.5 \mathrm{kpc}$ which exceeds the size of bright regions usually covered by a single burst of star formation. Abnormally moving gas is revealed by its Doppler velocities, which differ from the velocities of the surrounding disk or from the velocities expected for circular rotation at a given point. The velocity differences reach some tens of $\mathrm{km} \mathrm{s}^{-1}$, but sometimes they exceed $100 \mathrm{~km} \mathrm{~s}^{-1}$. Such regions are found in IC 1525, NGC 1084, NGC 2964, NGC 3893, and NGC 6643. These velocity anomalies are often accompanied by an appearance of secondary emission-line components which indicate the existence of at least two independent subsystems of ionized-gas clouds possessing normal and abnormal motions on the line of sight. Sometimes the presence of a "noncircular" velocity component is seen in the wide asymmetric wings of the emission line profiles. In IC 1525, NGC 1084, and partly in NGC 1134, the non-circular motions are 


\section{Galaxy: IC 1525}
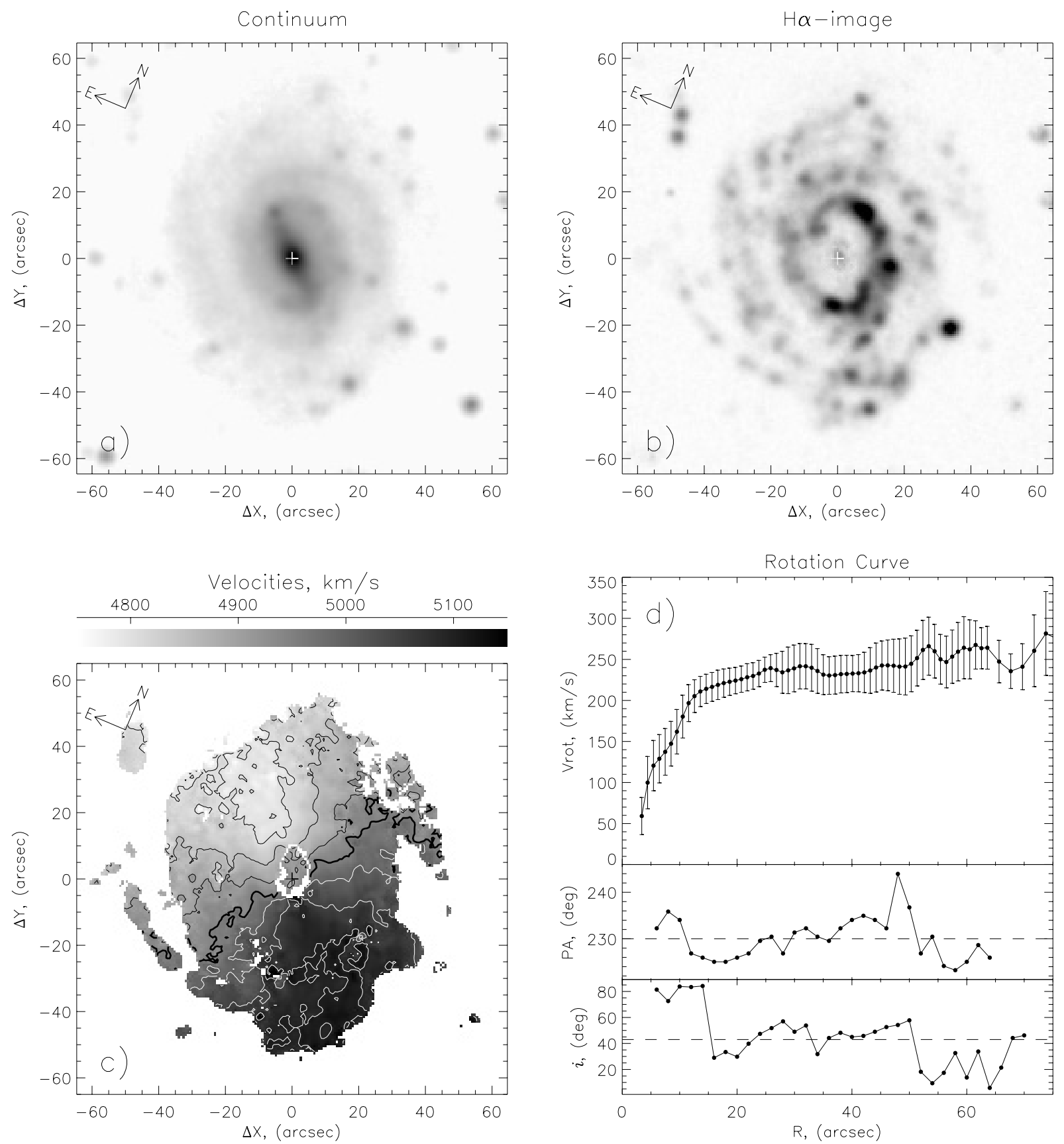

Fig. 5. IFP data for galaxy IC 1525. Top - image in continuum near $\mathrm{H} \alpha$ (left) and monochromatic image in $\mathrm{H} \alpha$ (right), a square-root scale is used. Bottom left - velocity field, the thickest black contour corresponds to the systemic velocity, other contours are $\pm 50, \pm 100, \pm 150 \ldots \mathrm{km} \mathrm{s}^{-1}$. A cross marks the dynamical centre. Bottom right - rotation curve $\left(V_{\text {rot }}\right)$, kinematic position angles (PA), and the inclination $(i)$ (both are obtained in a tilted-rings model). Dashed lines show the accepted kinematic parameters PA and $i$. The error-bars on the $V_{\text {rot }}(r)$ curve correspond to the mean non-circular velocities (see the text for details).

accompanied by an increase in the $[\mathrm{NII}] / \mathrm{H} \alpha$ ratio that argues in favor of a shock excitation mechanism (see the detailed discussion in Moiseev 2000).

The regions of these abnormal velocities are usually observed off the sites of active star formation, although the bright HII regions are often seen near them. It seems as if the gas with abnormal velocity is usually located between the complexes of HII. Nevertheless it does not mean that the "abnormal" gas definitely flows around normally rotating islands of star formation: indeed, the extended regions of this gas may cover bright HII regions in projection, which makes the emission of abnormally moving gas non-observable against the bright background.

These regions may be related to powerful "galactic fountains", where gas thrown out of star formation sites along the $z$ coordinate returns, falling toward the galactic disk (see for 
example Breitschwerdt \& Komossa 2000; de Alvis 2000). We observe this gas more easily when it is projected onto the areas of low gas emissivity. The other event which may account for the observed gas motion is the interaction of low density gas of a galactic disk with high velocity clouds accreting onto the galaxy. In this case, the appearance of star formation sites in this region is a consequence, not the cause, of the abnormal velocities of the gas. Further studies may distinguish between these possibilities.

\section{Conclusions}

The main results of this work may be summarized as follows:

- The presence of regular noncircular motion of gas related to spiral density waves may introduce systematic errors in the evaluation of the orientation parameters $i$ and $\mathrm{PA}_{0}$ of galactic disks. In particular, it affects the commonly used tilted-rings model. The systematic errors remain even if the parameters found for individual rings in this model are averaged. To avoid this effect, we force the orientation parameters to be constant all over the disk within some radius. Still, the tilted-rings model remains a useful diagnostic method of revealing non-coplanar or strongly non-axisymmetric gas motion at some distance from the center (in circumnuclear regions or the disk outskirts).

- For the sample of fifteen observed galaxies the parameters $i$ and $\mathrm{PA}_{0}$ were estimated using both the kinematic and photometric methods we describe in this work; the latter is based on the analysis of the second Fourier harmonic of azimuthal brightness distribution in the sky plane. Both kinematic and photometric methods of disk orientation parameter determination may have their limitations, especially in galaxies with strong triaxial (oval or bar) distortions. However, the close agreement of the orientation parameters found by two independent methods for most of our sample galaxies shows that the effect of systematic noncircular gas motion does not significantly alter the resulting parameters obtained from kinematic data for a pure circular rotation model.

- We have obtained the curves of rotation for 15 spiral galaxies, assuming the gas motion to be circular, and found the confidence intervals for rotation velocities at a given radii which characterize possible errors due to ignoring the regular non-circular motion related to spiral arms and/or a bar.

- Practically all the galaxies possess local non-circular gas motion, which appear either as residual velocities left after subtraction of circular velocities from the observed velocity fields, or as the complex profiles of emission lines, which may be decomposed into circular and non-circular components. Local anomalies of the velocity fields on a scale of several hundred pc (up to $1-1.5 \mathrm{kpc}$ ) are related to star formation or some other mechanisms, which are responsible for the non-circular velocities often exceeding $100 \mathrm{~km} \mathrm{~s}^{-1}$ (see the discussion in the previous section.)

- The observed regular large-scale non-circular motion in the galaxies have another nature: they are related to bars and/or spiral arms of galaxies. In principle, the detailed analysis of these density-wave-induced motions may allow to restore a full 3D vector velocity field of the gas in galactic disks (see the Introduction). This will be done as a future work.

Acknowledgements. The authors express their thanks to J. Boulesteix and S. Drabek for the software development used in the data processing and S. D. Ryder for the possibility to use IR images of two galaxies. We especially thank the referee, Dr. Y. Copin for his fruitful comments and remarks. We also appreciate the organizers of the HYPERCAT Database which was used in this paper. This research is partially based on data from the ING Archive and used the NASA/IPAC Extragalactic Database (NED) operated by JPL under contract with NASA. The authors also thank Max Fridman for improving the quality of the text.

This work was performed under partial financial support from RFBR grant N 02-02-16878, grant "Leading Scientific Schools" N 00-1596528 , and the contracts with Ministry of Industry, Science and Technology òò40.022.1.1.1101 and 40.020.1.1.1167.

\section{References}

Afanasiev, V. L., Burenkov, A. N., Zasov, A. V., \& Sil'chenko, O. K. 1988a, Afz, 28, 243

Afanasiev, V. L., Burenkov, A. N., Zasov, A. V., \& Sil'chenko, O. K. 1988b, Afz, 29, 155

Afanasiev, V. L., Burenkov, A. N., Zasov, A. V., \& Sil'chenko, O. K. 1991a, SvA, 35, 569

Afanasiev, V. L., Popravko, G. V., Sil'chenko, O. K., \& Zasov, A. V. 1991b, SvA Lett., 17, 325

Afanasiev, V. L., Burenkov, A. N., Zasov, A. V., \& Sil'chenko, O. K. 1992, SvA, 36, 10

Afanasiev, V. L., \& Sil'chenko, O. K. 1999, AJ, 117, 1725

Allsopp, N. J. 1979, MNRAS, 188, 371

Barth, A. J., Ho, L. C., Filippenko, A. V., \& Sargent, W. L. 1995, AJ, 110,1009

Amram, P., Boulesteix, J., Marcelin, M., et al. 1995, A\&AS, 113, 35

Beauvais, C., \& Bothun, G. 1999, ApJS, 125, 99

Beauvais, C., \& Bothun, G. 2000, ApJS, 128, 405

Beauvais, C., \& Bothun, G. 2001, ApJS, 136, 41

Begeman, K. G. 1989, A\&A, 223, 47

Bizyaev, D. V., Zasov, A. V., \& Kajsin, S. S. 2001, Astron. Lett., 27, 218

Bottinelli, L., Gouguenheim, L., Paturel, G., \& de Vaucouleurs 1984, A\&AS, 56, 381

Boulesteix, J. 1993, ADHOC Reference Manual (Marseille: Publ. de l'Observatoire de Marseille)

Breitschwerdt, D., \& Komossa, S. 2000, ApSS, 272, 13

Burlak, A. N., Zasov, A. V., Fridman, A. M., \& Khoruzhii, O. V. 2000, Astronomy Lett., 26, 809

Carignan, C., Sancisi, R., \& van Albada, T. S. 1988, AJ, 95, 37

Carollo, C. M., et al. 1997, AJ, 1114, 2366

Chapelon, S., Contini, T., \& Davoust, E. A\&A, 345, 81

Chevalier, R. A., \& Furenlid, I. 1978, ApJ, 225, 67

Contini, T., Considere, S., \& Davoust, E. 1998, A\&AS, 130, 285

De Jong, R. S. 1996, A\&AS, 118, 557

de Avilles, M. A. 2000, ApSpSci, 272, 23

De Robertis, M., \& Shaw, R. A. 1988, ApJ, 329, 629

Dodonov, S. N., Vlasyuk, V. V., \& Drabek, S. V. 1995, Fabry-Perot Interferometer. User's Guide, Nizhnii Arkhyz, in Russian

Frei, Z., Guhathakurta, P., \& Gunn, J. E. 1996, AJ, 111, 174

Elmegreen, D. M., \& Elmegreen, B. G. 1987, ApJ, 314, 3

Elmegreen, D. M., Chromey, F. R., Bissell, B. A., \& Corrado, K. 1999, AJ, 118, 2618 
Fridman, A. M., Khoruzhii, O. V., Lyakhovich, V. V., et al. 1997, Ap\&SS, 252, 115

Fridman, A. M., Khoruzhii, O. V., Zasov, A. V., et al. 1998, Astron. Lett., 24, 764

Fridman, A. M., Khoruzhii, O. V., Polyachenko, E. V., et al. 1999, Phys. Lett. A, 264, 85 [arXiv: astro-ph/0003097]

Fridman, A. M., \& Khoruzhii, O. V. 2003, Space Sci. Rev., 105, 1

Fridman, A. M., Khoruzhii, O. V., Lyakhovich, V. V., et al. 2001a, A\&A, 371, 538

Fridman, A. M., Khoruzhii, O. V., Polyachenko, E. V., et al. 2001b, MNRAS, 323, 651

Fridman, A. M., Khoruzhii, O. V., Minin, V. A., et al. 2001c, in Galaxy Disks and Disk Galaxies, ed. J. G. Funes, \& S. J. E. M. Corsini, San Francisco, ASP Conf. Ser., 230, 187

Fridman, A. M., Khoruzhii, O. V., \& Polyachenko, E. V. 2002, Observational Manifestation of Chaos in Astrophysical Objects (Kluwer acad. publ.), Space Sci. Rev., 102, 51

Garrido, O., Marcelin, M., Amram, P., \& Boulesteix, J. 2002, A\&A, 387,821

Garrido, O., Marcelin, M., Amram, P., \& Boissin, O. 2003, A\&A, 399, 51

Gonzalez, D. R. M., Perez, E., Tadhunter, C., et al. 1996, A\&AS, 115, 439

Grosbol, P. J. 1985, A\&AS, 60, 261

Ho, L. C., Fillipenko, A. V., \& Sargent, W. L. W. 1997, ApJS, 112, 315

Iye, M., Okamura, S., Hamabe, M., \& Watanabe, M. 1982, ApJ, 256, $103 \mathrm{I}$

Lyakhovich, V. V., Fridman, A. M., Khoruzhii, O. V., \& Pavlov, A. L. 1997, Astro. Rep., 41, 447

Mayya, Y. D., Ravindranath, S., \& Carrasco, L. 1998, AJ, 116, 1671

Moiseev, A. V. 2000, A\&A, 363, 843

Moiseev, A. V. 2002a, Preprint of SAO RAS, No. 163, 1

Moiseev, A. V. 2002b, Bulletin of the SAO, 54 [arXiv: astro-ph/0211104]

Moiseev, A. V., \& Mustsevoi, V. V. 2000, Astro. Lett., 26, 665 [arXiv: astro-ph/0011225]
Moiseev, A. V., Valdes, J.-R., \& Chavushyan, V. H. 2004, A\&A, 421, 433

Odenwald, S. F. 1986, ApJ, 320, 328

Pence, W. D. 1981, ApJ, 247, 473

Pogge, R. W. 1987, ApJS, 71, 433

Pustilnik, S. A., Kniazev, A. Y., Ugryumov, A. V., et al. 2001, Ap\&SS, 277, 109

Ravindranath, S., \& Prabnu, T. P. 1998, AJ, 115, 2320

Rogstad, D. H., Chu, K., \& Crutcher, R. M. 1979, ApJ, 229, 509

Rots, A. H., \& Shane, W. W. 1975, A\&A, 45, 25

Rubin, V. C., Ford, W. K., \& Thonnard, N. 1980, ApJ, 238, 471

Rush, B., \& Malkan, M. A. 1993, ApJS, 89, 1

Sakhibov, F. Kh., \& Smirnov, M. A. 1987, SvA, 31, 132

Sakhibov, F. Kh., \& Smirnov, M. A. 1989, SvA, 33, 476

Sakhibov, F. Kh., \& Smirnov, M. A. 1990, SvA, 34, 347

Sandage, A., \& Humphreus, R. M. 1980, ApJ, 236, L1

Schommer, R. A., Bothun, G. D., Williams, T. B., \& Mould, J. R. 1993, AJ, 105, 97

Sil'chenko, O. K., Vlasyuk, V. V., \& Alvarado, F. 2001, AJ, 121, 2499

Thornley, M. D. 1996, ApJ, 469, L45

Thornley, M. D., \& Mundy, L. G. 1997, ApJ, 490, 682

Tully, R. B., Verheijen, M. A. W., Pierce, M. J., et al. 1996, AJ, 112, 2471

Verheijen, M. A. W, 1996, Ph.D. Thesis

Warner, P. J., Wright, M. C. H., \& Baldwin, J. E. 1973, MNRAS, 163, 163

Wevers, B. M. H. R., van der Kruit, P. C., \& Allen, R. J. 1986, A\&AS, 66,505

Zasov, A. V., \& Moiseev, A. V. 1998, Astro. Lett., 24, 584

Zasov, A. V., \& Moiseev, A. 1999, in Activity in galaxies an related phenomena, ed. Terzian et al., IAU Symp., 194, ASP Conf. Ser., 279 [arXiv: astro-ph/9812121]

Zasov, A. V., \& Sil'chenko, O. K. 1987, SvAL, 13, 186

Zasov, A. V., \& Sil'chenko, O. K. 1996, in Barred Galaxies, ed. R. Buta, et al., IAU Coll., 157, ASP Conf. Ser., 91, 207 
A. M. Fridman et al.: The orientation parameters of spiral galaxies, Online Material $p 1$

\section{Online Material}




\section{Results for individual objects}

Figures illustrating the results obtained for all galaxies of our sample but the first one, IC 1525, are available in the electronic version of the paper.

\subsection{IC 1525}

Below we will use PA and $R$ as polar coordinates in the sky plane (where PA is the position angle measured from the North counterclockwise and $R$ is the distance from the galactic center).

The continuum image (Fig. 5a) reveals the presence of a prominent bar aligned at $\mathrm{PA}=48^{\circ}$. The bar ends are encircled by the ring of HII regions which has a radius of $15^{\prime \prime}-17^{\prime \prime}$ (Fig. 5b). In the central part of the bar, $R<5^{\prime \prime}-7^{\prime \prime}$, due to the superposition of strong absorption lines of bulge stars, the $\mathrm{H} \alpha$ emission line sinks in absorption, so the velocity field (Fig. 5c) has a hole in the center.

During the observation of this galaxy, the working spectral range of IFP contained not only $\mathrm{H} \alpha$, but also the emission line [NII] $\lambda 6583$ from the neighboring interference order, the most prominent line in the circumnuclear region (see Fig. 1b). We have tried to estimate the line-of-sight velocity of the ionized gas in the central region lacking the $\mathrm{H} \alpha$ emission by using the [NII] line, but the velocity field obtained in such a way deviates strongly from the simple extrapolation inward of the mean rotation field obtained from the $\mathrm{H} \alpha$ measurements further from the center. We think that the anomalous velocities obtained from the $[\mathrm{NII}]$ measurements in the region $r<5^{\prime \prime}$ may result from the impossibility to properly take into account the effect of the stellar absorption line $\mathrm{H} \alpha$ disturbing the profile of [NII] emission line because of the overlapping of interference orders. To treat the gas motion in the center of IC 1525 correctly, new observations of emission lines free of stellar absorptions are needed.

The residual velocity distribution, obtained by the subtraction of the mean rotation field from the observed velocity field, demonstrates several regions where a projection of non-circular velocities onto the line of sight exceeds $50 \mathrm{~km} \mathrm{~s}^{-1}$. The sizes of these regions corrected for the beam smearing are $1^{\prime \prime}-5^{\prime \prime}$ (up to $1.6 \mathrm{kpc}$ for the adopted distance of $67 \mathrm{Mpc}$ ). In the cylindrical coordinate frame on the sky plane, at the locus $R=28^{\prime \prime}$, $\mathrm{PA}=190^{\circ}$ the residual line-of-sight velocity is $50-80 \mathrm{~km} \mathrm{~s}^{-1}$; at the loci $\left(R=33^{\prime \prime}, \mathrm{PA}=34^{\circ}\right),\left(R=22^{\prime \prime}, \mathrm{PA}=105^{\circ}\right)$, and $\left(R=35^{\prime \prime}, \mathrm{PA}=75^{\circ}\right)$ the residuals have the opposite sign: $-(40-70) \mathrm{km} \mathrm{s}^{-1}$. It is interesting that the intensity ratio $[\mathrm{NII}] / \mathrm{H} \alpha$ is enhanced in these regions by a factor of $1.5-2$ with respect to the nearest outskirts of these local velocity anomalies (see Fig. 1a). At the locus $\left(R=26^{\prime \prime}, \mathrm{PA}=254^{\circ}\right)$ the relative intensity of [NII] is quite normal, but the width of the $\mathrm{H} \alpha$ emission line profile is $F W H M=200-220 \mathrm{~km} \mathrm{~s}^{-1}$, (after deconvolution with the instrumental contour), and the residual line-of-sight velocities are $-(50-80) \mathrm{km} \mathrm{s}^{-1}$. All five sites of anomalous velocities are located beyond bright HII regions and are not related spatially to the spiral arms.

A sharp steepening of the very outer part of the velocity curve in this and some other galaxies discussed below is evi- dently an artifact; the result of a small number of reliably measured points (often located near the minor axis of a galaxy) and/or of a bad agreement with a model of co-planar circular motion.

\subsection{NGC 23 (Mrk 545)}

The rotation curve of the galaxy (Fig. 6d) possesses a local maximum first noted by Afanasiev et al. (1988a). We have prolonged this rotation curve to the radius twice as large as that reached by Afanasiev et al. (1988a). Since at $r>30^{\prime \prime}$ there is only one bright "spiral arm" that contributes to the rotation velocity measurements, the local minimum of the rotation curve at $r \approx 40^{\prime \prime}$ is a possible artifact related to noncircular motion within this arm. The inclination of individual rings in a tilted-rings model vary strongly along the radius that evidently reflects significant deviations from circular motion of about 20-30 $\mathrm{km} \mathrm{s}^{-1}$, nevertheless the inclination $i$ and the position angle of line-of-nodes $\mathrm{PA}_{0}$ we found for this galaxy (see Table 3) remain to be consistent within their error with the earlier photometric and kinematic estimates (Afanasiev et al. 1988a). The $\mathrm{PA}_{0}$ of the kinematic major axis orientation in the inner part of NGC 23 also coincides within a few degrees with the estimate of Afanasiev et al. (1991b) made with the integral field (multipupil) spectrograph MPFS.

The problem of possible bar presence in NGC 23 remains unsolved. According to RC3, the galaxy is classified as SB, and de Jong (1996), by applying a 2D decomposition method to its brightness map, finds a bar with a length of $29^{\prime \prime}-32^{\prime \prime}$ aligned along PA $=154^{\circ}$. But the velocity field obtained by us does not demonstrate any noticeable turn of the kinematic major axis (Figs. 6c and 6d), and there is also no sign of any isovelocity twisting when passing from the outer to the inner region of the galaxy (Afanasiev et al. 1991b). The local small-scale twisting of the isovelocities at $r=5^{\prime \prime}-8^{\prime \prime}$ (Fig. 6d) is rather related to a small (mini- ) bar with a length of about $10^{\prime \prime}$, which is implied by the CCD- observations of Chapelon et al. (1999).

In the circumnuclear region $\left(r<5^{\prime \prime}\right)$ the emission line $\mathrm{H} \alpha$ is broadened up to $F W H M=200-300 \mathrm{~km} \mathrm{~s}^{-1}$, which was already noted by De Robertis \& Shaw (1988) from their highresolution spectroscopy. Their Fig. 1 demonstrates two local maxima of $F W H M$ located symmetrically with respect to the nucleus at $r=6^{\prime \prime}-8^{\prime \prime}$. Our IFP spectra of these spots reveal strong either red or blue wings of the $\mathrm{H} \alpha$ line profiles. The Gauss analysis of these profiles allows us to derive "non-circular" velocity components shifted with respect to the main component by $130-200 \mathrm{~km} \mathrm{~s}^{-1}$ at $\left(R=6^{\prime \prime}, \mathrm{PA}=323^{\circ}\right)$ and by $-(170-200) \mathrm{km} \mathrm{s}^{-1}$ at $\left(R=3^{\prime \prime}, \mathrm{PA}=190^{\circ}\right)$. The profiles of the emission lines in these regions are shown in Figs. 1c and 1d. The estimated diameters of these regions are $3^{\prime \prime}-4^{\prime \prime}$, being close to our spatial resolution element. We should note that it is at the location of the "blue" component where Afanasiev et al. (1991b) had found non-circular motion of the ionized gas with velocities of $260-300 \mathrm{~km} \mathrm{~s}^{-1}$, but the asymmetry of the emission line profiles was missed by them because of their low spectral resolution. The same authors have argued that NGC 23 may be at the post-Seyfert stage. In this 
case the anomalous gas motion may be related to the activity, or to a starburst in the nucleus. But the emission line intensity ratios in the nucleus of NGC 23 evidence rather in favor of HII-region character of excitation and are not consistent with the hypothesis of Sy2 or LINER (De Robertis \& Shaw 1988; Contini et al. 1998). Since the sites of the anomalous velocities are located at the ends of a nuclear minibar (see above), the presence of the second velocity component may be related to non-circular motion in the bar. But in this case one needs to explain a very high degree of non-circular motion - more than $100 \mathrm{~km} \mathrm{~s}^{-1}$ in projection along the line of sight.

\subsection{NGC 615}

In the circumnuclear region $\left(r<10^{\prime \prime}-15^{\prime \prime}\right)$ the broad absorption line $\mathrm{H} \alpha$ is superposed on the emission line [NII] $\lambda 6548$ from the neighboring interference order that prevents an exact determination of the line-of-sight velocities even by a Gaussian analysis. We start our measurements beyond the radius of the circumnuclear local rotation velocity maximum (at $r \approx 5^{\prime \prime}$ ) detected by Afanasiev et al. (1988a). Beyond $r=10^{\prime \prime}$ the relative intensity of the nitrogen emission lines falls, so they do not affect the profile of $\mathrm{H} \alpha$ any more.

The most prominent feature of the monochromatic $\mathrm{H} \alpha$ image (Fig. 7b) is a pair of HII regions located symmetrically with respect to the nucleus at the radius of $20^{\prime \prime}-23^{\prime \prime}$. They are slightly elongated along the radius and look like shock fronts at the edges of a bar (though the galaxy is classified as unbarred). At the same distance from the center the gas velocity field is disturbed by non-circular motion resulting in a turn of the kinematic major axis by more than $20^{\circ}$ at $r=15^{\prime \prime}-30^{\prime \prime}$. We have found here several spots with the line-of-sight velocities differing by $40-50 \mathrm{~km} \mathrm{~s}^{-1}$ from the mean rotation field. These kinematic details may be evidence for a triaxial potential within $r=25^{\prime \prime}-30^{\prime \prime}$ in this galaxy. Indeed, the recent photometric analysis of Sil'chenko et al. (2001) has revealed that NGC 615 possesses a separate inner compact disk of oval shape especially notable at $r=15^{\prime \prime}-30^{\prime \prime}$ where it introduces nonmonotonic changes of isophote orientation and ellipticity.

\subsection{NGC 972}

The near-H $\alpha$ continuum image (Fig. 8a) lacks a spiral structure, but on the $\mathrm{H} \alpha$ image (Fig. $8 \mathrm{~b}$ ) the HII regions concentrate toward a pseudo-ring with the radius of about $30^{\prime \prime}$. Several works which analyze CCD-data on NGC 972 (Ravindranath \& Prabnu 1998; Zasov \& Moiseev 1998) also contain remarks about the absence of a noticeable spiral structure on the broad-band optical images and about a large amount of dust within the ring of HII regions. However, near-infrared images obtained through $J H K$-filters reveal a prominent two-armed spiral pattern and a small bar with the size of $10^{\prime \prime}$ elongated in $\mathrm{PA}=175^{\circ}-180^{\circ}$ (Mayya et al. 1998; Zasov \& Moiseev 1998). This peculiarity makes the morphological classification of the galaxy very ambiguous; e.g. Mayya et al. (1998) classify it as SABdpec while according to NED (RC3) it is Sab.
The isovelocities of the ionized-gas velocity field within the central $10^{\prime \prime}$ have a characteristic S-shape (Fig. 8c), evidently induced by a presence of the bar seen in NIR. A turn of the kinematic major axis in the center exceeds $30^{\circ}$ (Fig. 8d); the noncircular (radial?) gas streams in the bar are so significant that a cross-section of the velocity field in $\mathrm{PA} \approx 30^{\circ}$ gives an impression of counterrotation. We should note here that the presence of a minibar in the center of NGC 972 had been first claimed by Zasov \& Sil'chenko (1996) from kinematic arguments.

The orientation parameters found by us for the outer part of the disk (Fig. 8d) are consistent within the errors with both photometric (Ravindranath \& Prabnu 1998; Zasov \& Moiseev 1998) and kinematic results from our earlier long-slit observations (see Zasov \& Moiseev 1998; who used the data from Afanasiev et al. 1991a). The rotation curve derived in this work also agrees rather well with that obtained by Zasov \& Moiseev (1998).

Ravindranath \& Prabnu (1998) noted a high rate of star formation in the central part of NGC 972, comparable even to that of the well-known starburst galaxies M 82 and NGC 253. We see indirect consequences of this intense star formation when analyzing our Fabry-Perot spectra: many HII regions stand out by their negative residual velocities of about -(15-20) $\mathrm{km} \mathrm{s}^{-1}$, which is probably related to expanding bubbles of ionized gas. A negative sign of residuals may be accounted for by the presence of internal absorption, dimming the radiation of receding parts of the bubbles. In the nucleus, at $r<2^{\prime \prime}-3^{\prime \prime}$, which is close to our spatial resolution, the $\mathrm{H} \alpha$ emission-line profile has mostly a two-component structure; it can be related either to gas outflow from the starburst nucleus or to non-circular gas motion at the edges of the minibar (analogous to NGC 1084, see below).

\subsection{NGC 1084}

The emission line [NII] $\lambda 6583$ from the neighboring interference order is seen throughout the galaxy; it has allowed us to study the kinematics of the ionized gas by using two emission lines separately instead of a single $\mathrm{H} \alpha$. From the earlier observations by Afanasiev et al. (1988a), two regions with strong velocity deviations from the mean rotation were found. Particularly in the central region, $r<5^{\prime \prime}$, the rotation velocities obtained by measuring the forbidden emission lines [NII] and [SII] were systematically lower than those obtained by measuring $\mathrm{H} \alpha$. The authors had interpreted this peculiarity as evidence for minibar presence in the center of NGC 1084. Besides that, non-circular motions were found at the periphery of the galaxy, at $r=40^{\prime \prime}-50^{\prime \prime}$ to the north-east from the center, near an elongated arc-like spur outgoing from the spiral arm near a giant superassociation (Fig. 9b).

Our IFP data confirms the existence of these kinematically distinct regions. Near the spur, the profile of the $\mathrm{H} \alpha$ emission line is mostly two-component: one component traces the mean rotation and the other is shifted by $\pm 100- \pm 150 \mathrm{~km} \mathrm{~s}^{-1}$. The appearance of the non-circular component is supplemented by a sharp increase of the $[\mathrm{NII}] / \mathrm{H} \alpha$ ratio by a factor of $2-6$ with respect to the normal one in the neighboring HII regions. The 
areas of non-circular motion have typical sizes of 1-2 kpc and are located between the HII regions. A possible interpretations of these non-circular motions of ionized gas near the spur is discussed elsewhere (Moiseev 2000).

In the circumnuclear region, $r<5^{\prime \prime}$, the profiles of the $\mathrm{H} \alpha$ and $[\mathrm{NII}]$ emission lines also demonstrate a two-component structure, with the velocity separation of the components about $50 \mathrm{~km} \mathrm{~s}^{-1}$ (Figs. 1e and 1f). The Gaussian analysis of the $\mathrm{H} \alpha$ profile has allowed us to trace azimuthal variations of the observed velocity gradients for each component. We have found that the brighter component traces the circular rotation of the ionized gas with the kinematic major axis close to the major axis of the galaxy, whereas the fainter component of both lines corresponds to the gas radial motion because its projected velocity gradient is zero near the line of nodes.

In the distance range of $5^{\prime \prime}-20^{\prime \prime}$ on the opposite sides of the nucleus, in the direction $\mathrm{PA} \approx 60^{\circ}$, the extended linear front is revealed. At both sides of this front the character of asymmetry of the $\mathrm{H} \alpha$ and [NII] profiles changes abruptly: the red wings are replaced by blue ones. The line-of-sight residual velocities of the main line components change their sign across the front jumping by $80-100 \mathrm{~km} \mathrm{~s}^{-1}$. The width of the front is $3^{\prime \prime}-4^{\prime \prime}$, so it remains practically unresolved. Such a combination of features evidences that we are dealing with supersonic gas flow in a strong shock front.

Features similar to those observed in NGC 1084 (radial gas flows, narrow shock fronts) are usually treated as a result of bar influence; but neither our continuum image of NGC 1084 (Fig. 9a), nor broad-band photometric images of the galaxy (Zasov \& Moiseev 1999; Moiseev 2000) reveal any signatures of a bar. We should also note that the turn of the kinematic major axis in the radius range of the shock fronts $\left(5^{\prime \prime}-20^{\prime \prime}\right)$ is rather small (Fig. 9d), which implies the absence of a high contrast triaxial potential.

A whole complex of peculiarities observed in the center of NGC 1084 may be interpreted as a result of a weak triaxial potential (a small triaxial bulge). Similar shock fronts at the edges of a slightly triaxial bulge have been also observed in Sb galaxy NGC 2841 by Afanasiev \& Sil'chenko (1999), but in that galaxy the bulge is much more massive.

\subsection{NGC 1134}

Due to its complex inner structure (multiple dust filaments, etc.) the galaxy is included in Arp's Atlas of Peculiar Galaxies (1966), though it is not an interacting system. In the area $r<20^{\prime \prime}-30^{\prime \prime}$, HII regions trace a three-arm spiral (Fig. 10b), but in the outer part of the galaxy the spiral pattern is two-armed. The three-arm spiral structure in the center of NGC 1134 can also be seen when analyzing its direct images by constructing maps of a color Q-parameter free of dust influence (Bizyaev et al. 2001).

The mean orientation of the line of nodes of the disk (Fig. 10d) is consistent with the earlier photometric and kinematic estimates (Afanasiev et al. 1991b), though our estimate of the inclination is significantly lower than $i=72^{\circ}$ used by these authors. At small radii, $r<12^{\prime \prime}$, the kinematic major axis (Fig. 10d) turns abruptly by more than $15^{\circ}$, so that the isovelocities (Fig. 10c) demonstrate an S-like shape typical for a bar potential. Above all, on both sides of the nucleus, at $r=4^{\prime \prime}-7^{\prime \prime}$ there are regions where $\mathrm{H} \alpha$ emission-line profiles are two-component, with a separation between the components of $150-200 \mathrm{~km} \mathrm{~s}^{-1}$ (see Figs. $10 \mathrm{~g}$ and $10 \mathrm{~h}$. These regions of the two-component $\mathrm{H} \alpha$ emission line are elongated along the direction of $\mathrm{PA} \approx 20^{\circ}-30^{\circ}$ and resemble the shock edges in NGC 1084. Close to these shock fronts, at the minor axis of the galaxy, one can note two spots where the secondary velocity component disguises itself as an asymmetric wing of the $\mathrm{H} \alpha$ emission-line profiles, and the velocities obtained for the peak of profiles differ from the mean rotation velocities by $\pm(80-100) \mathrm{km} \mathrm{s}^{-1}$. Since the anomalous velocities are observed near the minor axis of the galaxy, they may be explained as the presence of radial gas flows. We should mention that for the first time the regions of the non-circular gas motion on both sides of the nucleus in NGC 1134 were detected by Afanasiev et al. (1991b).

All the kinematic peculiarities of the central region of NGC 1134 mentioned above can be explained by the hypothesis of a bar aligned with the minor axis of the galaxy.

The mean rotation curve calculated by us (Fig. 10d) agrees with the one reported earlier by Afanasiev et al. (1991b): the solid-body area up to $r=7^{\prime \prime}$, a low-contrast peak, and after that a velocity plateau. At $r=65^{\prime \prime}-75^{\prime \prime}$ and at $r=90^{\prime \prime}-110^{\prime \prime}$ there are two emission "islands" in the disk, emerging by $70-90 \mathrm{~km} \mathrm{~s}^{-1}$ from the extrapolated flat velocity of rotation, which are responsible for the sharp jumps in the calculated velocity curve at large distances from the center. These anomalous details can be related either to a gas-disk orientation change at large radii (a "warp"), or to the possibility that these HII regions do not belong to the global disk plane, being dwarf satellites of NGC 1134. To clarify these possibilities, the results of HI observations of the outer disk kinematics are needed.

\subsection{NGC 2964}

The galaxy possesses a well-developed two-arm spiral structure; HII regions at $r=30^{\prime \prime}-35^{\prime \prime}$ are concentrated to a pseudoring (Figs. 11a and 11b). It has a close companion, NGC 2968, at the distance of 5.8, but there are no evident signatures of interaction of galaxies.

At $r=1^{\prime \prime}-5^{\prime \prime}$ the kinematic major axis (Fig. 11d) turns abruptly by more than $15^{\circ}$, which can be assumed to be the effect of a small bar (note that there is a remark about the "very faint bar" in RC1). The high-resolution image of the central $18^{\prime \prime} \times 18^{\prime \prime}$ region of NGC 2964 obtained by HST and published by Carollo et al. (1997) reveals the presence of a complex pattern of dust filaments tracing a two-arm spiral and penetrating into the center of the galaxy. Similar dust spirals were found by HST imagers in the centers of many galaxies during the last years (e.g. Martini \& Pogge 1999; Regan \& Mulchaey 1999; Barth et al. 1995).

There is a region between the nucleus and a spiral arm, at $r=5^{\prime \prime}-12^{\prime \prime}$ to the south of the nucleus, where $\mathrm{H} \alpha$ emission-line profiles are clearly two-component 
(Fig. 1i). The main line component traces the mean rotation; the secondary component is redshifted by $\sim 150 \mathrm{~km} \mathrm{~s}^{-1}$, sometimes being as strong as the main one. At the outskirts of this region the $\mathrm{H} \alpha$ profiles often possess a red wing, so at the residual velocity map the whole area located between bright HII regions is distinguished by strong non-circular motion reaching $50-70 \mathrm{~km} \mathrm{~s}^{-1}$. The diameter of the anomalous area is $10^{\prime \prime}-15^{\prime \prime}$ $(1-1.5 \mathrm{kpc}$ for the adopted galaxy distance of $18 \mathrm{Mpc})$. As this area is located at the galactic minor axis, the velocity residuals will be caused by radial gas motions if they are confined to the plane of the galactic disk.

\subsection{NGC 3583}

This galaxy is a member of the pair of galaxies shared with NGC 3577 (their separation is $5^{\prime}$ ), but there is no clear evidence of interaction. A small elliptical companion may be found at a distance of about 1' (see the note in RC2 catalog). The continuum image of the galaxy reveals the presence of a bar which can be traced up to $r \approx 30^{\prime \prime}$ and is aligned with $\mathrm{PA}=80^{\circ}$; it also contains a lot of HII regions (Figs. 12a and $12 \mathrm{~b}$ ). Isovelocities close to the bar have a typical S-like shape (Fig. 12c), the kinematic major axis turns here by more than $20^{\circ}$, which is evidence of a significant contribution of noncircular motion into the line-of-sight velocity field (Fig. 12d). Indeed, within the bar, in the radius range of $2^{\prime \prime}-18^{\prime \prime}$, the lineof-sight velocities differ from the mean rotation velocity field by $\pm(50-80) \mathrm{km} \mathrm{s}^{-1}$. The "blue" and "red" residual velocity spots are located symmetrically on both sides of the center, almost along the minor axis of the galactic disk, which enables us to propose a radial gas flow due to the bar. The presence of a broad stellar $\mathrm{H} \alpha$ absorption line in the circumnuclear region of this galaxy introduces some uncertainty into the line-of-sight velocity estimates, but in comparison to other analogous cases (IC 1525, NGC 4414, etc.), this effect is not as severe.

\subsection{NGC 3893}

The galaxy forms a pair with NGC 3896 without any sign of tidal interaction (their separation is 3'.9). The rotation curve rises all along the radius, having no clear maximum (Fig. 13d). The turn of the kinematic major axis at $r<20^{\prime \prime}$ is related to the velocity field perturbation caused by a small bar aligned at $\mathrm{PA} \approx 20^{\circ}$, which is barely seen in the continuum image (Fig. 13a). The variations of $\mathrm{PA}_{0}$ at $r=20^{\prime \prime}-50^{\prime \prime}$ may be a spiral arm effect. At the radii of $37^{\prime \prime}-45^{\prime \prime}$ in the direction of PA $=140^{\circ}$, at the inner edge of the spiral arm, the $\mathrm{H} \alpha$ emission-line profiles have a double-peaked structure (see Fig. 1j), the secondary (non-circular) component being redshifted by $+50 \mathrm{~km} \mathrm{~s}^{-1}$. The region where the secondary velocity component appears has a diameter of $8^{\prime \prime}-9^{\prime \prime}$ (about $500 \mathrm{pc}$ for the adopted distance of $13 \mathrm{Mpc}$ ), is located between bright $\mathrm{HII}$ regions, and is related to local dynamical processes in the gaseous disk rather than to the spiral arms.

The orientation angles of this galaxy were also estimated from the $\mathrm{H} \alpha$ velocity field by Garrido et al. (2002), by minimizing the dispersion of the points along the rotation curve.
The value found by them, $\mathrm{PA}_{0}=347^{\circ}$, is close to the $345^{\circ} \pm 2^{\circ}$ accepted in this paper, but the difference of inclination angles $\left(30^{\circ}\right.$ versus $\left.45^{\circ} \pm 5^{\circ}\right)$ is significant. Note however that the accuracy of measurements of $i$ given by Garrido et al. (2002) is rather low, $\pm\left(5^{\circ}-10^{\circ}\right)$.

\subsection{NGC 4100}

This galaxy is a member of the Ursa Major cluster (Odenwald 1986), although it has no noticeable neighbors - the distance to the nearest luminous cluster galaxy is no less than $210 \mathrm{kpc}$ (see the map of the cluster in Tully et al. 1996). Both CCD images and surface brightness profiles reveal a central "lens" between the radii of $15^{\prime \prime}$ and $70^{\prime \prime}$ containing a pair of tightly wound spiral arms. Beyond the "lens", the surface brightness falls abruptly, although some interesting details - particularly two low-contrast symmetric arms - can still be seen in the outer parts of the disk.

Our IFP data covers only the region of the central "lens"; here the $\mathrm{H} \alpha$ emission is confined to the spiral arms and to the very bright circumnuclear region, $r<12^{\prime \prime}$ (Fig. 14b). According to Pogge (1987), this circumnuclear region concentrates more than $20 \%$ of the total $\mathrm{H} \alpha$ emission of the galaxy. The emission-line intensity ratios in the nuclear spectrum are typical for HII regions (Ho et al. 1997), hence a high $\mathrm{H} \alpha$ brightness of the nucleus may be attributed to intense star formation burst, distinguished in the background of the more quiescent galactic disk.

A kinematic study of this galaxy's ionized gas with a longslit spectrograph (Afanasiev et al. 1988b, 1992) has shown that the circumnuclear region of NGC 4100 is dynamically decoupled from the rest of the disk: its rotation curve demonstrates two local extrema at $r= \pm 5^{\prime \prime}$, with a rotation velocity gradient of about $300 \mathrm{~km} \mathrm{~s}^{-1} \mathrm{kpc}^{-1}$ between them, which may be evidence of a highly concentrated bulge (Afanasiev et al. 1988b). Above all, Afanasiev et al. (1992) noted that the kinematic major axis in the central region, $r<5^{\prime \prime}-6^{\prime \prime}$, was turned by about $25^{\circ}$ with respect to the outer isophote orientation. The authors treated this fact as a result of minibar influence in the center of this galaxy.

Our analysis of the velocity field of $t$ he ionized gas in NGC 4100 (Fig. 14c) confirms the dynamical decoupling of the circumnuclear region, although the local maximum of the rotation curve (Fig. 14d) is not as prominent as it appeared followed from Afanasiev et al. data. Besides the turn of the kinematic major axis, from $\mathrm{PA}_{0}=350^{\circ} \pm 2^{\circ}$ for the outer galaxy to $358^{\circ} \pm 3^{\circ}$ for the inner one (Fig. $14 \mathrm{~d}$ ), the inclination determined from the kinematics in the tilted-rings model changes abruptly from $i=72^{\circ} \pm 2^{\circ}$ for the outer galaxy to $i=60^{\circ} \pm 5^{\circ}$ for the inner one. As it was noted in the third section of this paper, the change of $i$ in a tilted-rings model may be just a reflection of the ordered non-circular coplanar motion of a gas. In general, presence of a bar may easily imitate the inclination variations in a line-of-sight velocity field (Moiseev \& Mustsevoi 2000). But in this particular galaxy, the photometric major axis turns in the same direction as the kinematic one, so we cannot exclude the presence of an inclined gas disk in the circumnuclear re- 
gion of NGC 4100 (Zasov \& Moiseev 1999). The radius of this disk is less than $11^{\prime \prime}-12^{\prime \prime}(0.8 \mathrm{kpc}$ for the adopted distance of $16 \mathrm{Mpc}$ ). Depending on which side of the circumnuclear disk is nearest to us, the inclination of the central disk to the global galactic plane must be either $25^{\circ}$ or $55^{\circ}$.

Our rotation curve for NGC 4100 (Fig. 14d) demonstrates a monotonic rise of the rotation velocity up to the spatial limits of the measurements, which is consistent with HI observations of Verheijen (1996), demonstrating that the rotation velocity rises up to $r=90^{\prime \prime}$; in the very outer part of the galaxy the rotation velocity reaches a plateau.

\subsection{NGC 4136}

According to Allsopp (1979), the galaxy is a possible member of the CVnII group. In the central part $\left(r<15^{\prime \prime}\right)$ there is a small bar weakly recognizable in our continuum image (Fig. 15a), but clearly seen on the CCD images given in The Digital Atlas of Nearby Galaxies by Frei et al. (1996). The galaxy is seen almost face-on, and as a result, the parameters $i$ and $\mathrm{PA}_{0}$ are poorly determined (see the dispersion of the estimates in Table 3). For this reason, the absolute value of the rotation velocity is rather uncertain. The "troughs" at the rotation velocity (Fig. 15d) may be just artifacts. Formally, this uncertainty is expressed by large error bars in Fig. 15. Under such a disk orientation, the line-of-sight velocity deviations from the mean rotation in most cases may be related mostly to gas motion along $z$-coordinate direction.

At the radii of $4^{\prime \prime}-15^{\prime \prime}$ the $\mathrm{H} \alpha$ emission is either negligible, or strongly contaminated by the absorption line due to bulge stars; this effect cannot be properly taken into account because of the IFP interference order overlapping.

\subsection{NGC 4414}

According to Elmegreen \& Elmegreen (1987), NGC 4414 is a flocculent galaxy; our continuum image (Fig. 16a) does not show any traces of a spiral pattern either. However, observations in the NIR bands reveal the presence of several extended segments of spiral arms populated by old stars (Thornley 1996). In the circumnuclear region of NGC 4414 we see a narrow $\mathrm{H} \alpha$ emission line inside the broad absorption produced by a contribution of bulge stars; within several arcseconds from the galactic center only the [NII] emission line from the neighboring order of interference is seen (Fig. 1k). But unlike other similar cases (IC 1525, NGC 615, etc.), for this galaxy we have been able to construct a velocity field of the ionized gas in the central region by subtracting the absorption line superposed and by applying a Gaussian analysis to the $\mathrm{H} \alpha$ and [NII] emission-line profiles. As a result, only a small area with a size of 7 " to the south-east of the nucleus remained uncovered by our measurements (Fig. 16c). Our orientation parameters for NGC $4414\left(\mathrm{PA}=156^{\circ}, i=56^{\circ}\right)$ are very close to those obtained by Thornley \& Mundy (1997) from the analysis of the HI and $\mathrm{CO}$ velocity fields $\left(\mathrm{PA}=160^{\circ}, i=60^{\circ}\right)$. The HI velocity field measured in this work extends beyond three optical radii of the galaxy and demonstrates a strong asymmetry of the outer disk at $r>200^{\prime \prime}$, although no optical satellite is found within the nearest outskirts of NGC 4414 (Thornley \& Mundy 1997).

The turn of the kinematic major axis and large error bars of the mean rotation velocity estimates at $r<9^{\prime \prime}$ (Fig. 16d) may be caused by non-circular gas motion in the minibar area. The presence of a minibar in NGC 4414 was first suspected by Thornley \& Mundy (1997) from isovelocity twisting seen in the HI velocity field, though the spatial resolution of the HI observations was much worse than our resolution of the ionized gas observations.

\subsection{NGC 4814}

The main fraction of the $\mathrm{H} \alpha$ emission in this galaxy is confined to the inner disk having a radius of $50^{\prime \prime}$; beyond this radius the emission regions are concentrated in a pair of spiral arms (Fig. 13b). A mean rotation curve (Fig. 17d) agrees well with the rotation curve of the inner region, $r<30^{\prime \prime}$, we obtained from the earlier long-slit observations at the $6 \mathrm{~m}$ telescope (Zasov \& Sil'chenko 1987; Afanasiev et al. 1988b). A turn of the kinematic major axis at $r<15^{\prime \prime}$ may be related to a small bar, but it is not accompanied by the significant continuum isophote turn. Afanasiev et al. (1988b) reported noncircular gas motion to the west of the nucleus, at $r \approx 20^{\prime \prime}$. After subtracting the mean rotation field from our velocity data, we have found that the residual velocities in this region are about $-40 \mathrm{~km} \mathrm{~s}^{-1}$, evidently produced by an effect of the spiral pattern on the kinematics of the gaseous disk.

\subsection{NGC 5371}

This galaxy possesses a modest bar of about $20^{\prime \prime}-30^{\prime \prime}$ length aligned with the minor axis of the galactic disk. The bar is barely seen on our continuum image (Fig. 18a), but reveals itself rather well on the other optical images (e.g. Gonzalez et al. 1996). Unfortunately the IFP observations of this galaxy were made under poor seeing conditions during a non-photometric night. Due to this, we present velocity measurements only for the brightest emission regions in the spiral arms (Fig. 18c). In the circumnuclear region the $\mathrm{H} \alpha$ emission line is strongly broadened, and the intense [NII] emission line from the neighboring interference order is overlaid (the [NII] emission is abnormally strong because NGC 5371 is classified as a LINER, Rush et al. 1993). We have not succeeded in separating the contributions of the overlapping broad emission lines into the integrated profile. Perhaps due to this uncertainty our measurements of the line-of-sight gas velocities in the circumnuclear region do not reveal the local rotation velocity maximum at $r=10^{\prime \prime}-15^{\prime \prime}$ reported by Zasov \& Sil'chenko (1987). It is worth noting that the inclination angle $i$ we obtained from the velocity field, $i=47^{\circ} \pm 8^{\circ}$, is somewhat larger than $i=34^{\circ}$, adopted by Wevers et al. (1986).

\subsection{NGC 6643}

According to the classification of Elmegreen \& Elmegreen (1987), the galaxy has a 5th arm class, and therefore is almost 
lacking an ordered spiral structure. Indeed, both our continuum and $\mathrm{H} \alpha$ images (Figs. 19a and 19b) present a flocculent-like galaxy. However, recent NIR observations by Elmegreen et al. (1999) reveal the presence of a two-arm spiral pattern in NGC 6643 related to an old stellar population and implying a dynamical origin. Afanasiev et al. (1988b) noted large-scale non-circular gas motion in the central part of the galactic disk of this galaxy. Our analysis of the velocity field shows that line-of-sight velocity deviations from the pure rotation field do exist, but they do not exceed $40-50 \mathrm{~km} \mathrm{~s}^{-1}$ over the whole disk of the galaxy. These motions are probably related to the spiral structure; but an exact diagnosis will be possible only after a detailed Fourier analysis of the velocity field, which is under way. However there are a few regions in the galaxy where the $\mathrm{H} \alpha$ emission-line profile cannot be fitted by a single Gaussian function. For example, at $r=12^{\prime \prime}$ to the north-west of the nucleus, between bright HII regions, the $\mathrm{H} \alpha$ profiles demonstrate a broad red wing that may be evidence of the presence of the secondary "non-circular" velocity component of $100-200 \mathrm{~km} \mathrm{~s}^{-1}$ in projection on the line of sight (see Fig. 11). The size of this anomalous velocity area is $5^{\prime \prime} \times 10^{\prime \prime}$ (about $1 \mathrm{kpc}$, under the adopted distance of $19 \mathrm{Mpc}$ ). The effect of this local velocity anomaly on the whole velocity field is rather strong: it is responsible for a turn of the kinematic major axis observed in the radius range of $r=3^{\prime \prime}-12^{\prime \prime}$ (Fig. 19d). If we mask this region when analyzing the velocity field, the position angle of the kinematic major axis in the central region becomes about $40^{\circ}$, in good agreement with the orientation of the line of nodes of the outer disk (Table 3 ). We should also note that the inclination $i \approx 60^{\circ}$ found in this work is less than $i=68^{\circ}$, used by Afanasiev et al. (1988b) but agrees with $i=59^{\circ}$ of Bottinelli et al. (1984) obtained from photometric data. 
A. M. Fridman et al.: The orientation parameters of spiral galaxies, Online Material $p 8$

\section{Galaxy: NGC23}
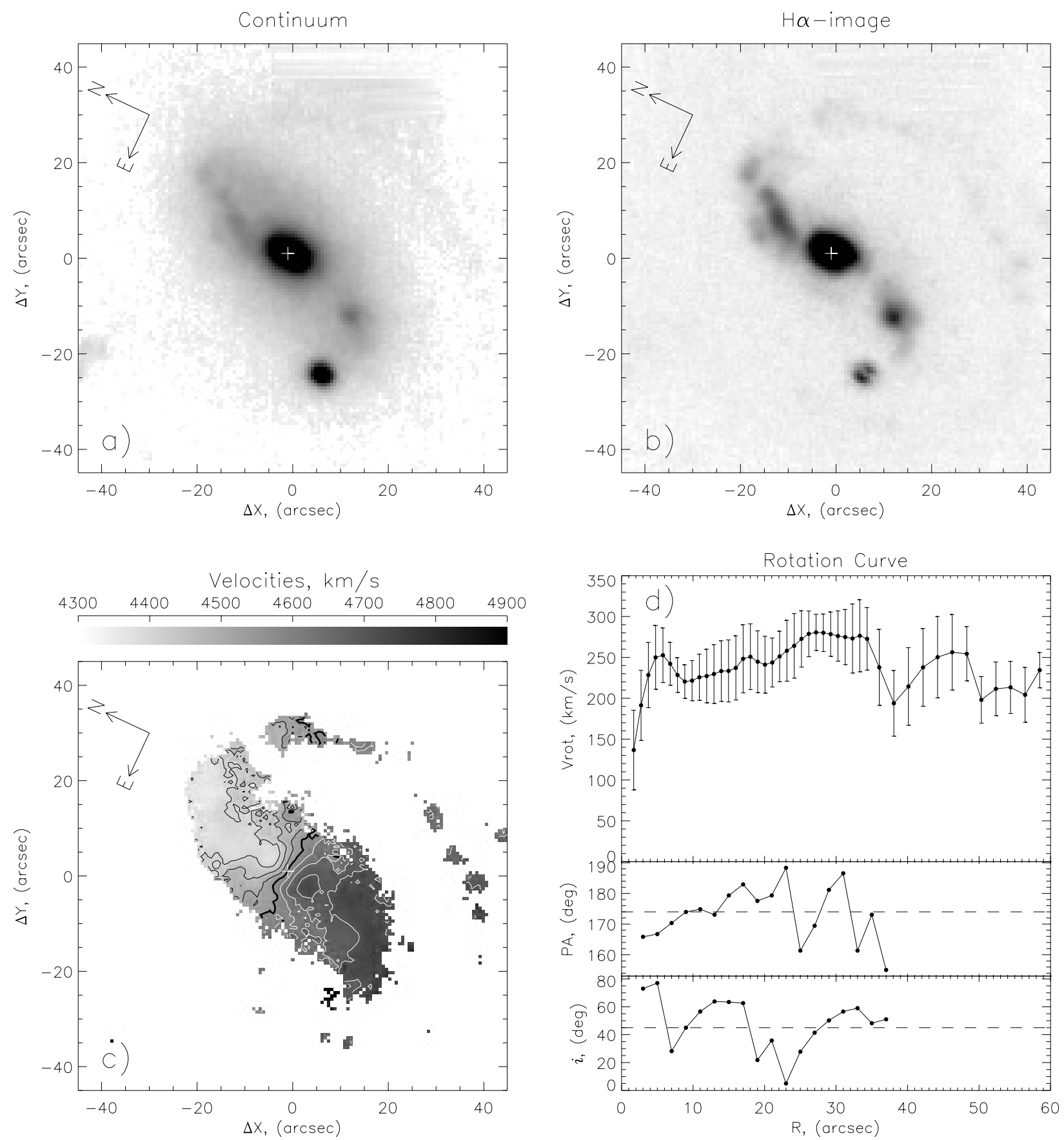

Fig. 6. The same as Fig. 5 for NGC 23. 
A. M. Fridman et al.: The orientation parameters of spiral galaxies, Online Material $p 9$

\section{Galaxy: NGC615}
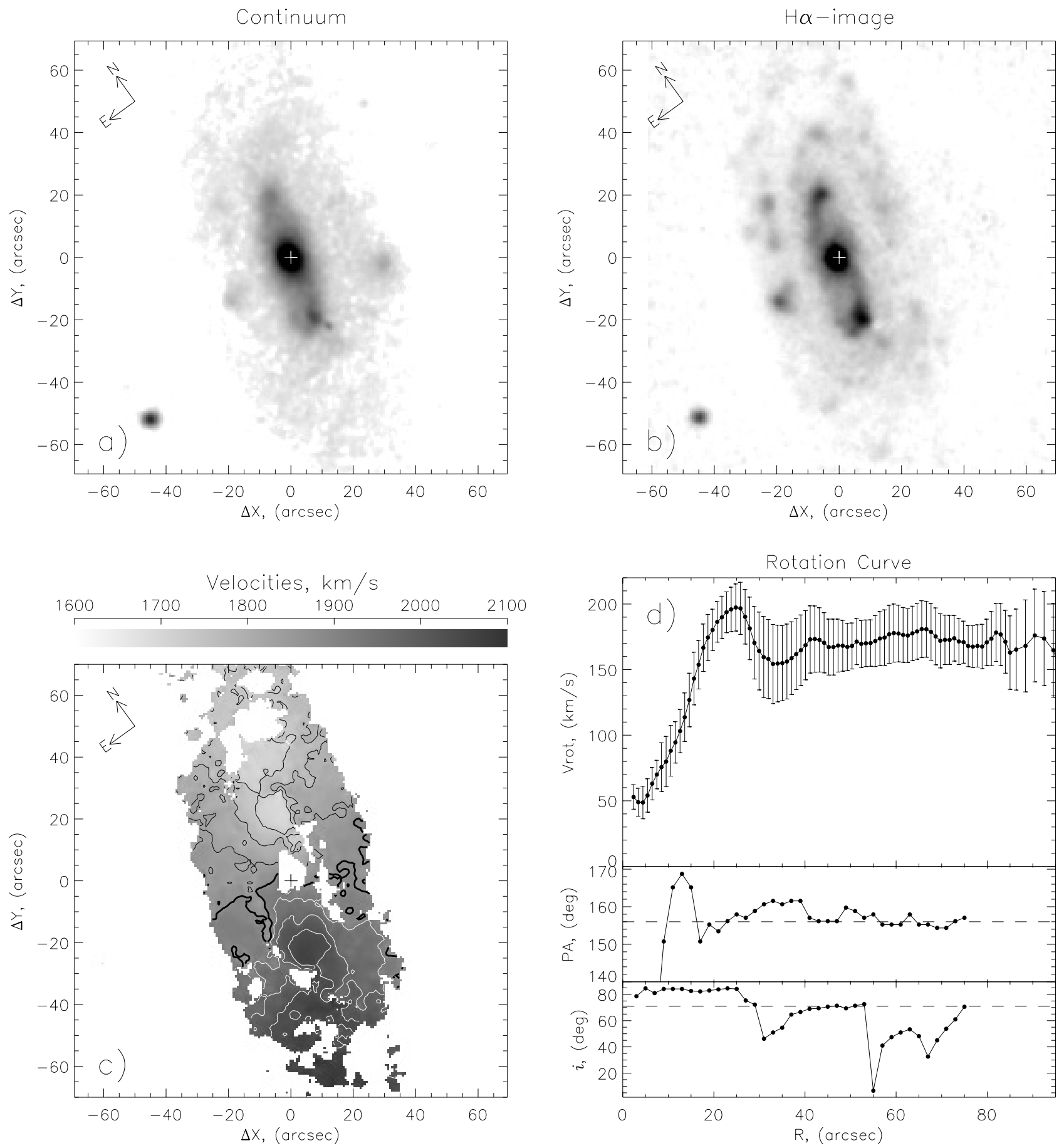

Fig. 7. The same as Fig. 5 for NGC 615. 
A. M. Fridman et al.: The orientation parameters of spiral galaxies, Online Material p 10

\section{Galaxy: NGC972}
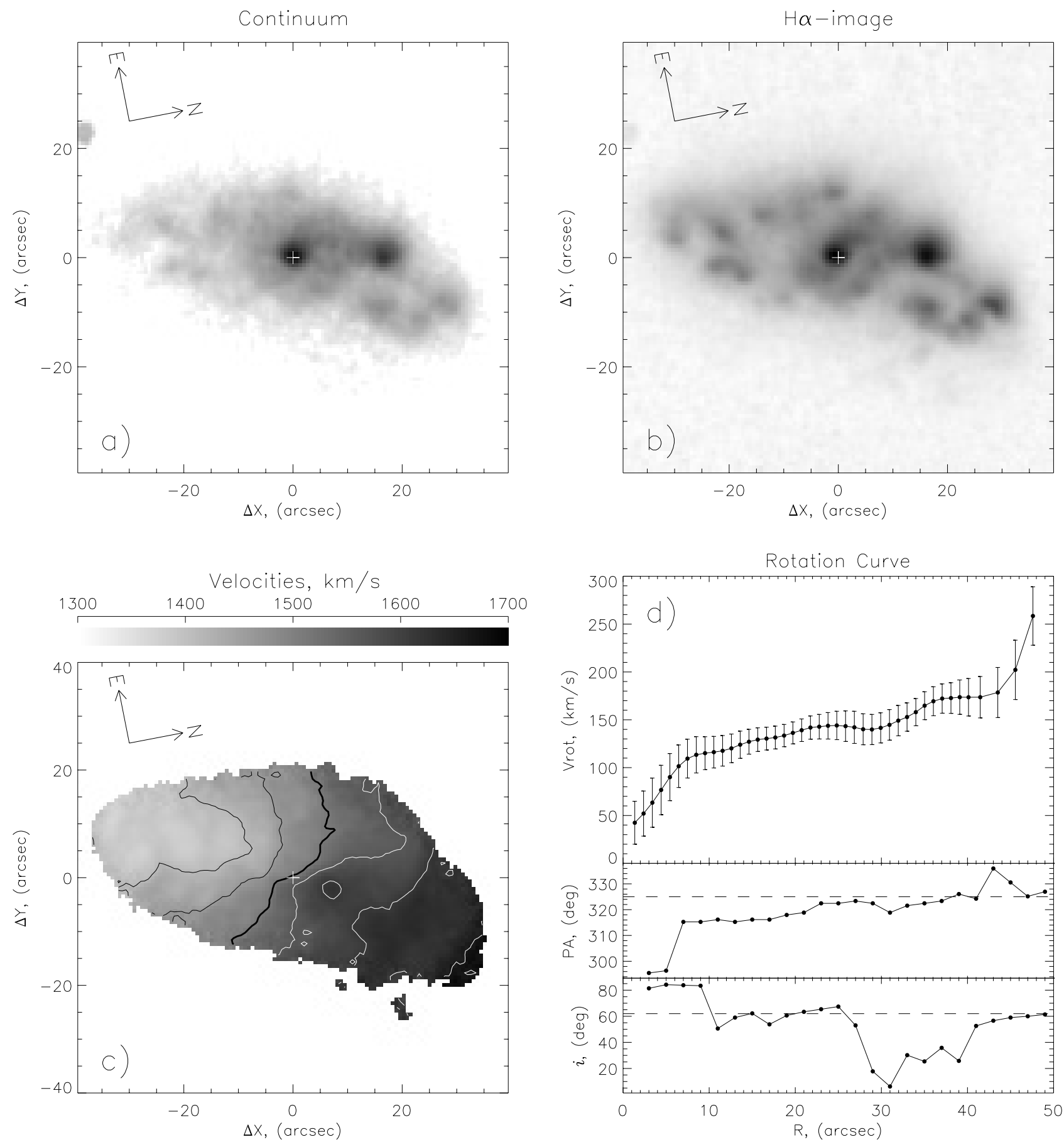

Fig. 8. The same as Fig. 5 for NGC 972. 
A. M. Fridman et al.: The orientation parameters of spiral galaxies, Online Material p 11

\section{Galaxy: NGC1084}
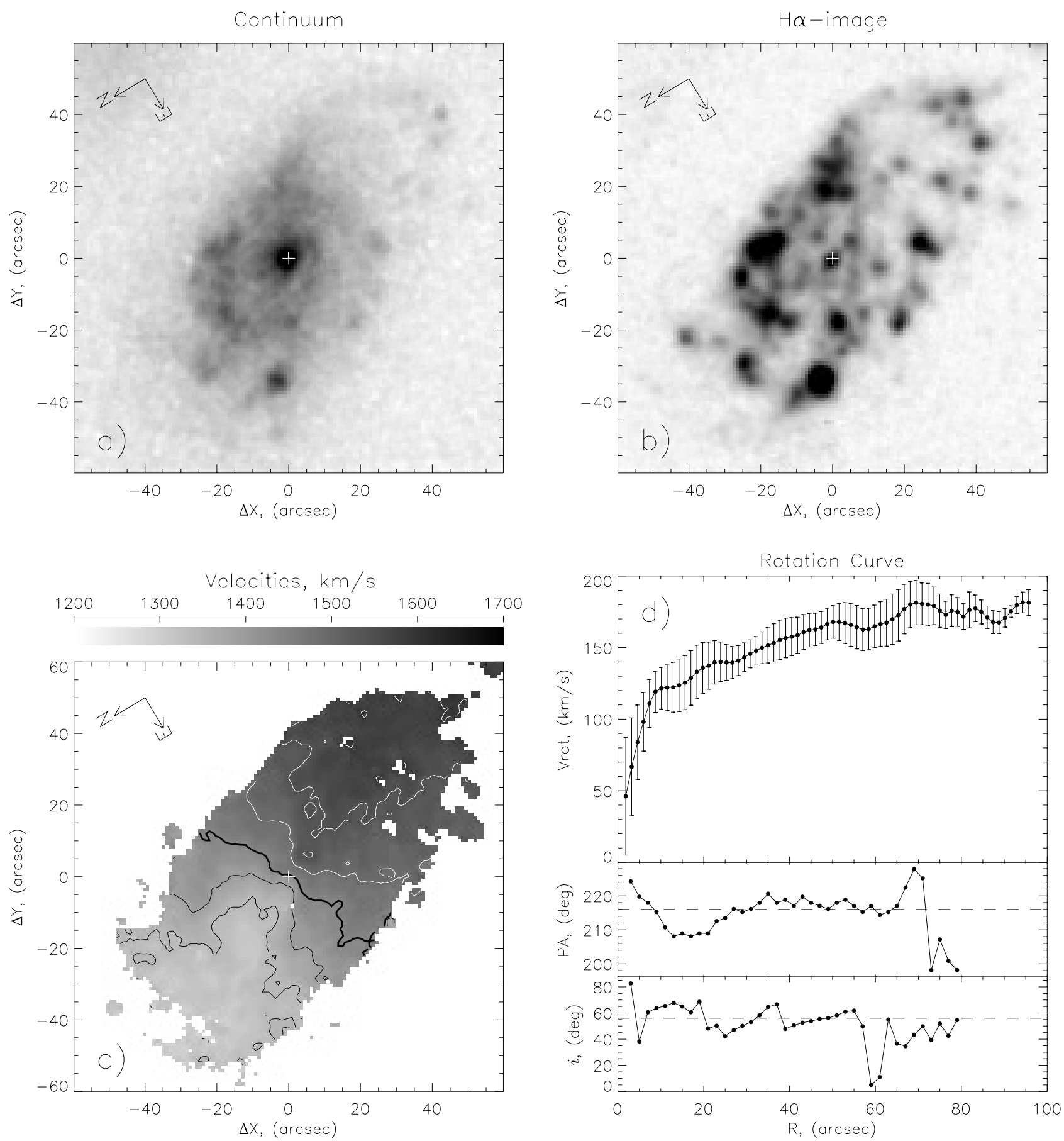

Fig. 9. The same as Fig. 5 for NGC 1084. 
A. M. Fridman et al.: The orientation parameters of spiral galaxies, Online Material p 12

\section{Galaxy: NGC1134}
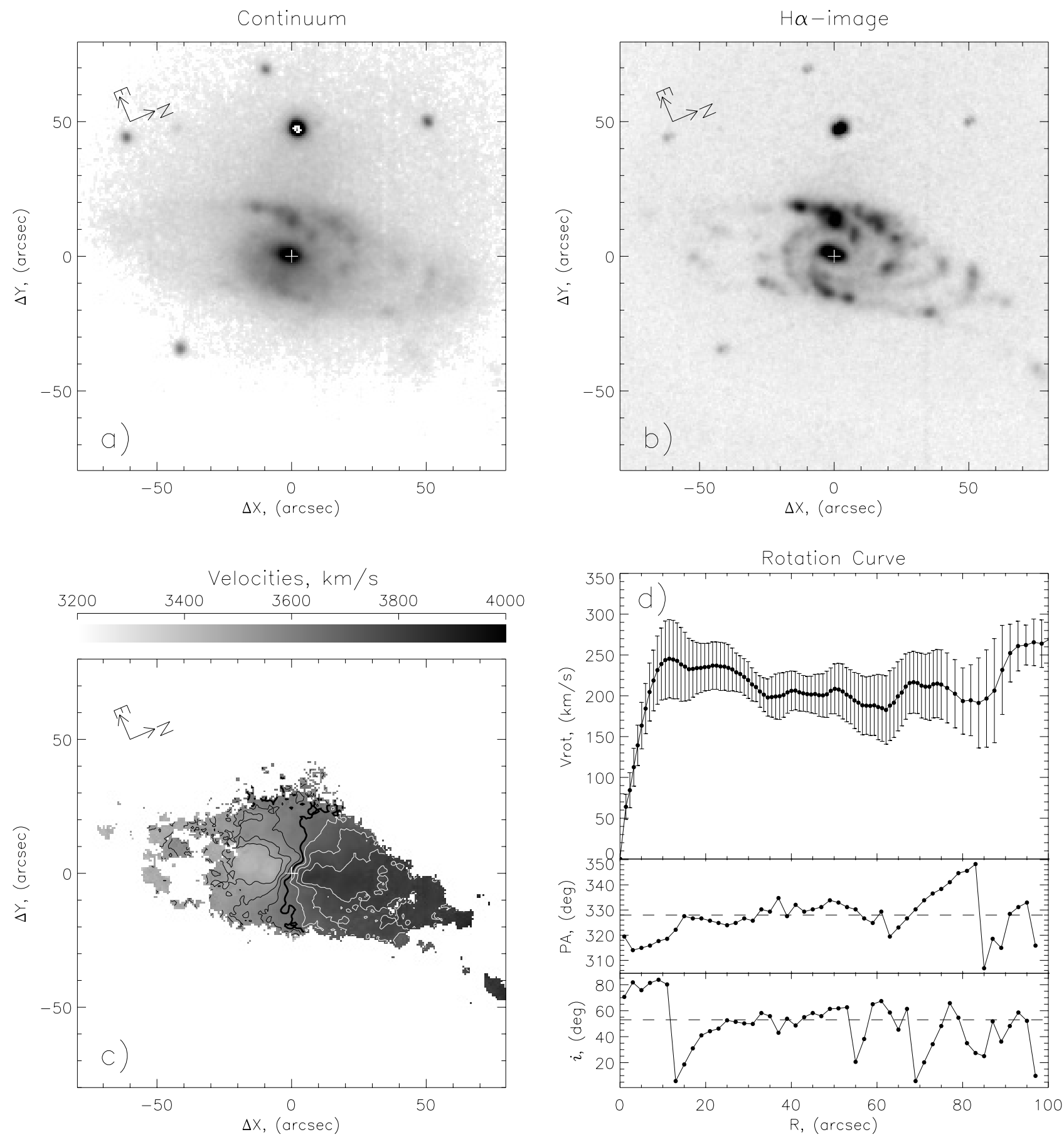

Fig. 10. The same as Fig. 5 for NGC 1134. 
A. M. Fridman et al.: The orientation parameters of spiral galaxies, Online Material p 13

Galaxy: NGC2964
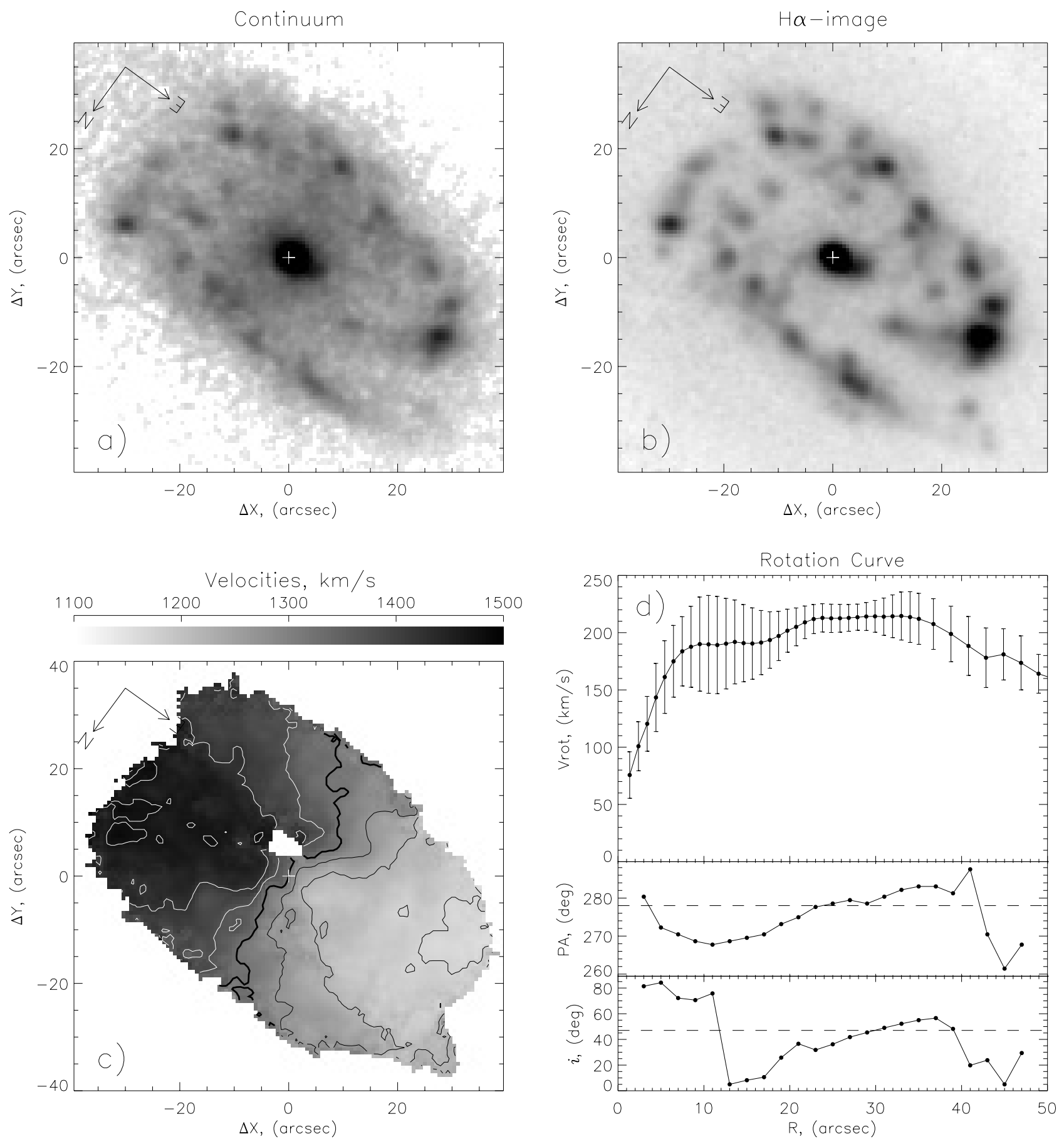

Fig. 11. The same as Fig. 5 for NGC 2964. 
A. M. Fridman et al.: The orientation parameters of spiral galaxies, Online Material p 14

\section{Galaxy: NGC3583}
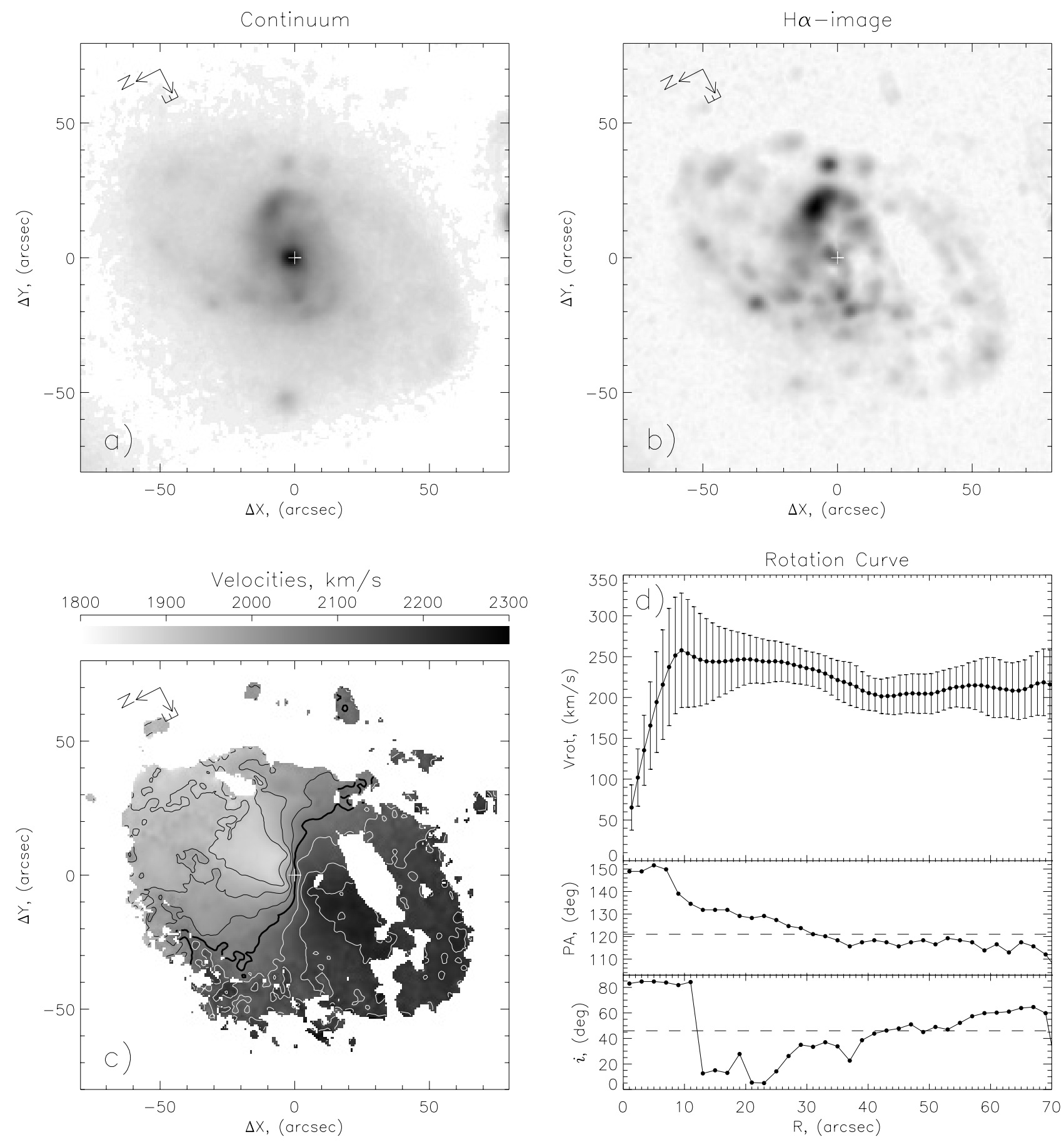

Fig. 12. The same as Fig. 5 for NGC 3583. There is a ghost-image in the bottom-left corner of the monochromatic and continuum images. 
A. M. Fridman et al.: The orientation parameters of spiral galaxies, Online Material p 15

Galaxy: NGC3893
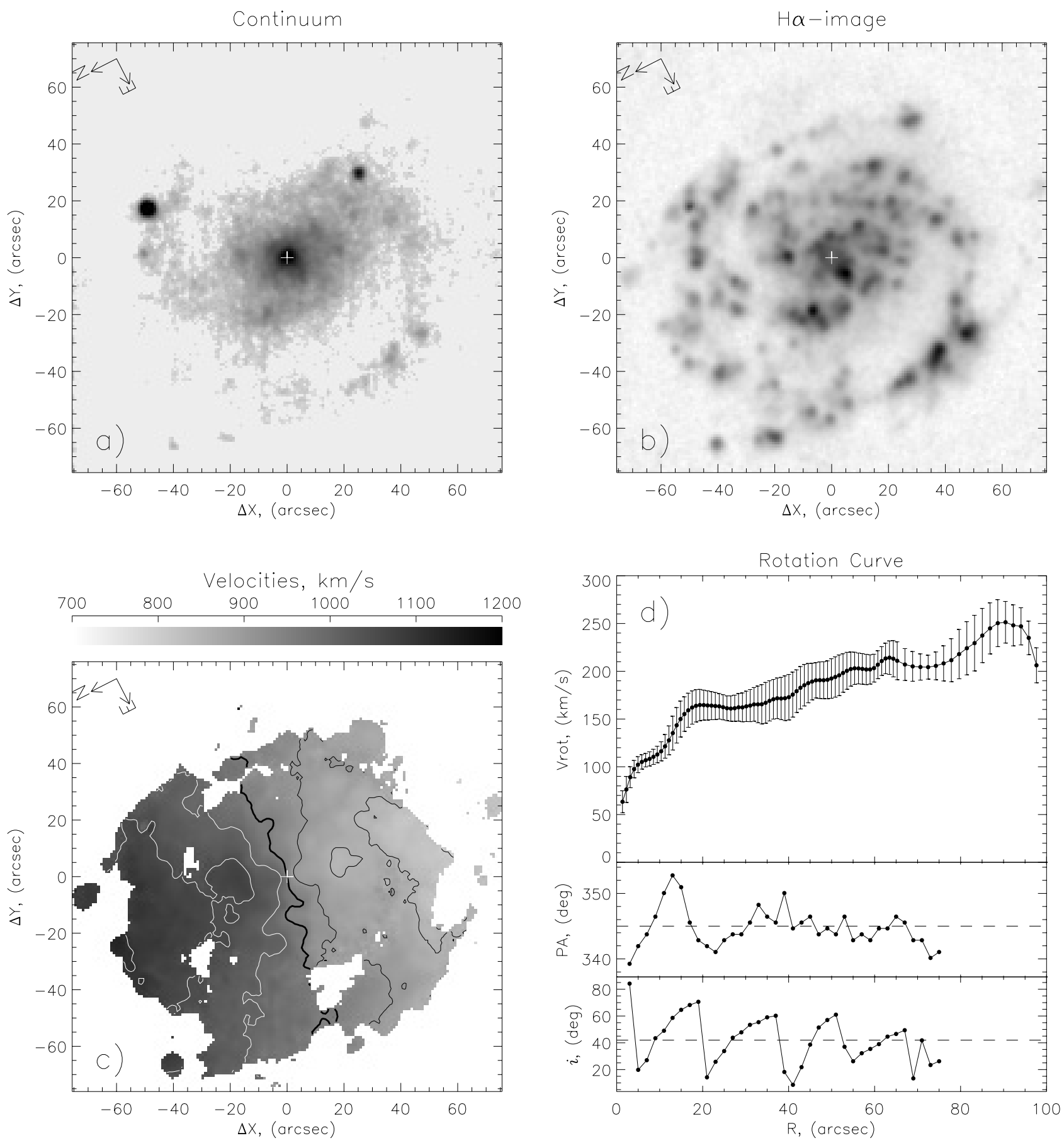

Fig. 13. The same as Fig. 5 for NGC 3893. 
A. M. Fridman et al.: The orientation parameters of spiral galaxies, Online Material p 16

\section{Galaxy: NGC4100}
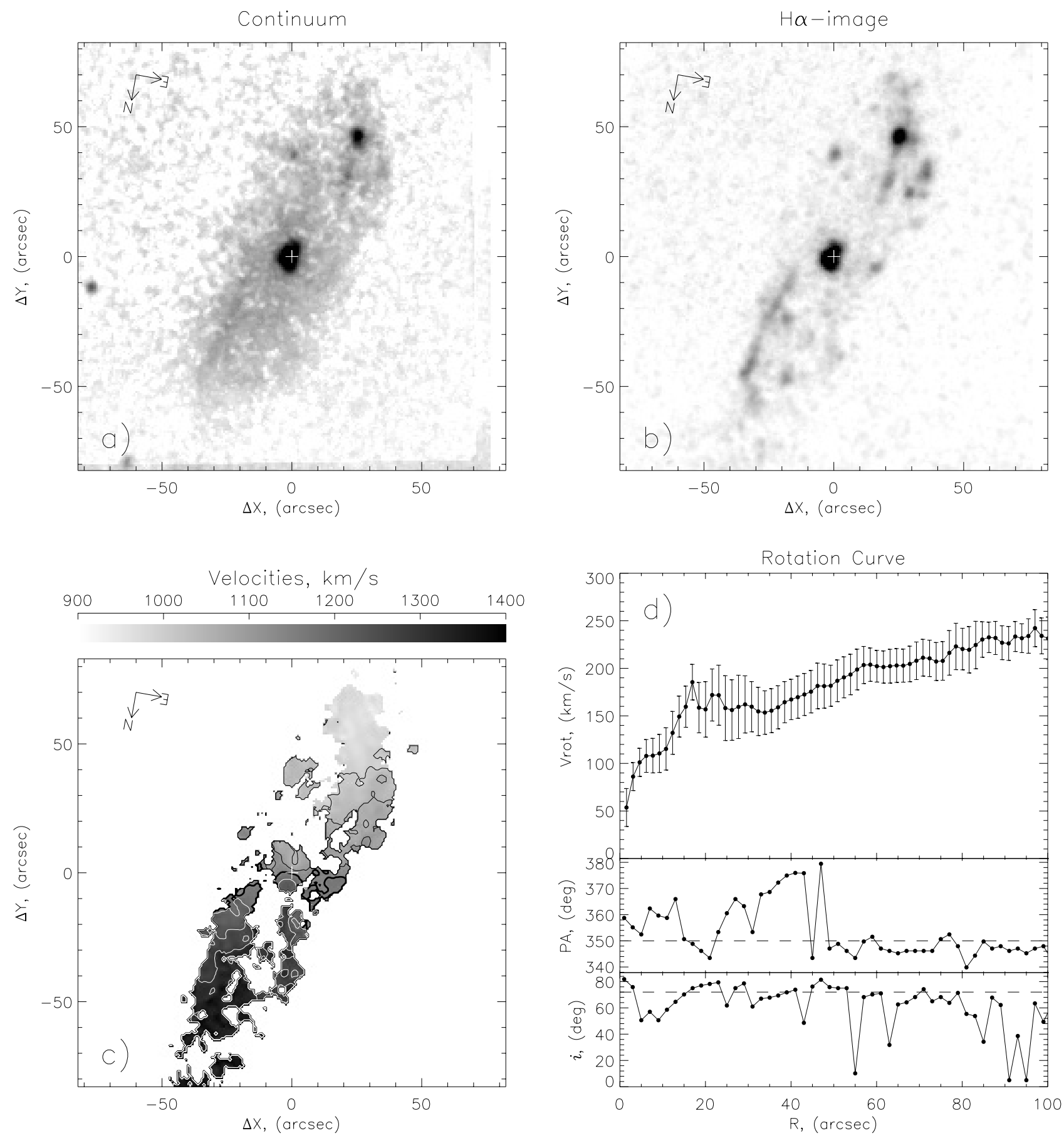

Fig. 14. The same as Fig. 5 for NGC 4100. 
A. M. Fridman et al.: The orientation parameters of spiral galaxies, Online Material p 17

\section{Galaxy: NGC4136}
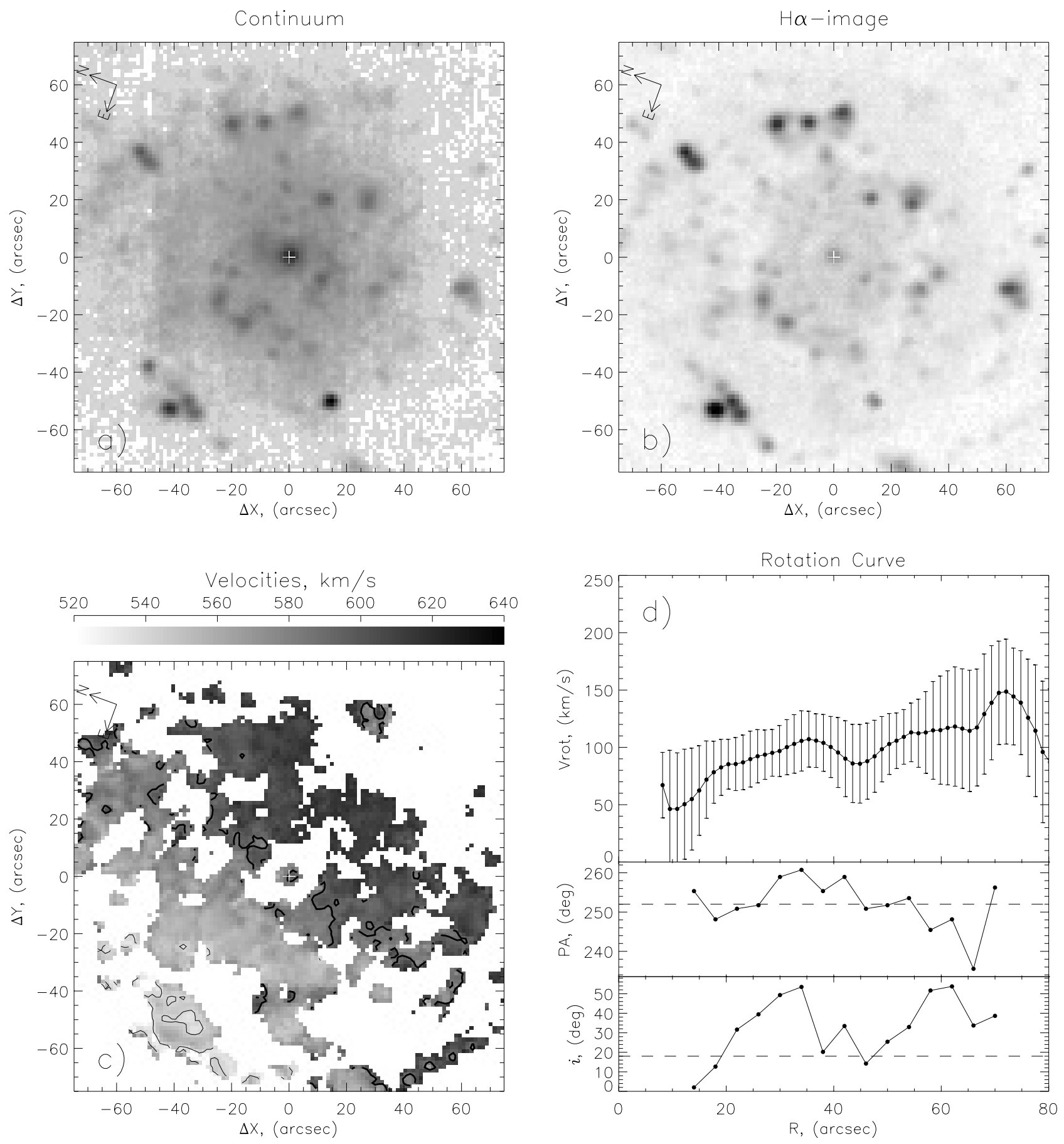

Fig. 15. The same as Fig. 5 for NGC 4136. 
A. M. Fridman et al.: The orientation parameters of spiral galaxies, Online Material p 18 Galaxy: NGC4414
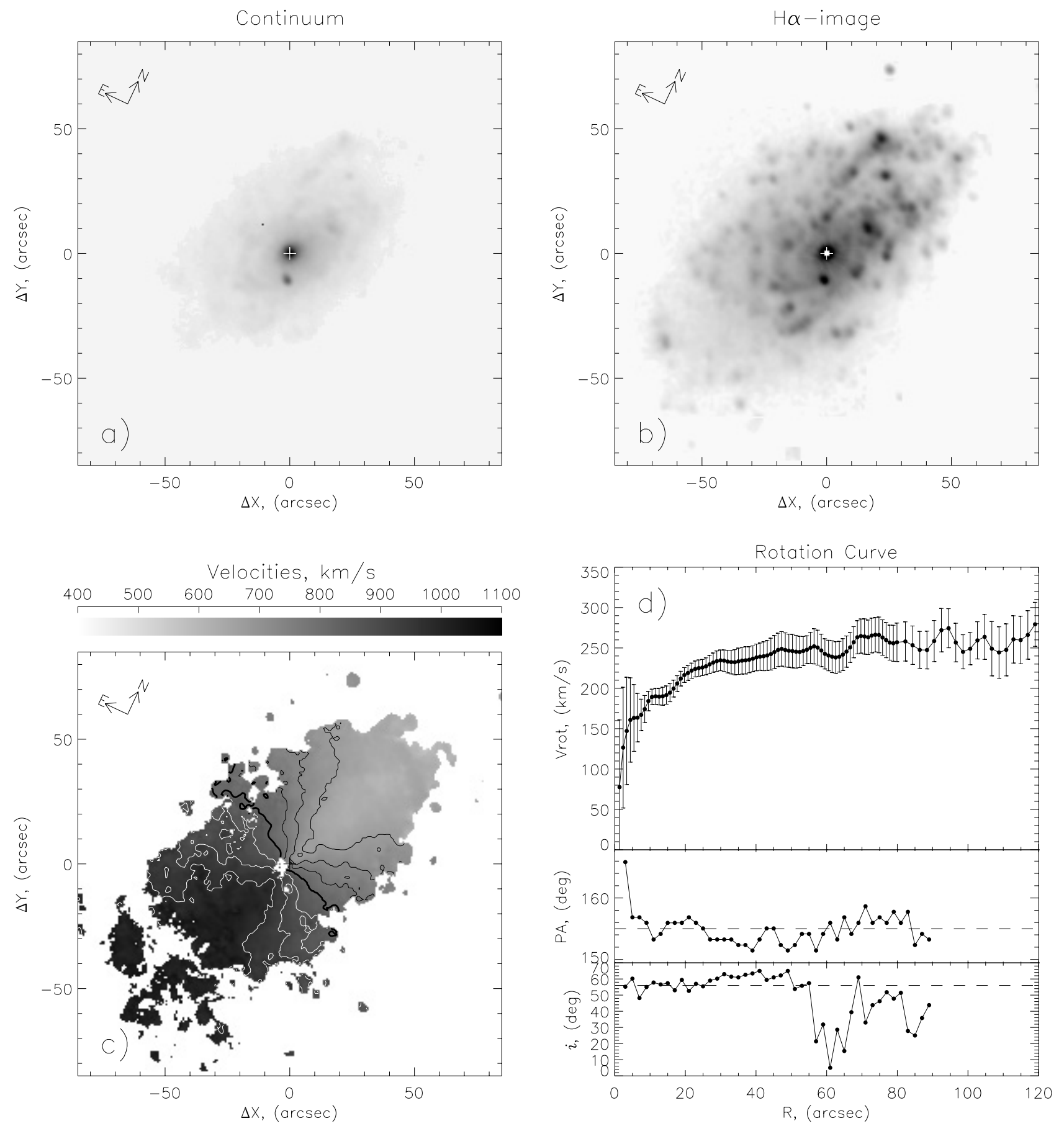

Fig. 16. The same as Fig. 5 for NGC 4414. 
A. M. Fridman et al.: The orientation parameters of spiral galaxies, Online Material p 19

\section{Galaxy: NGC4814}
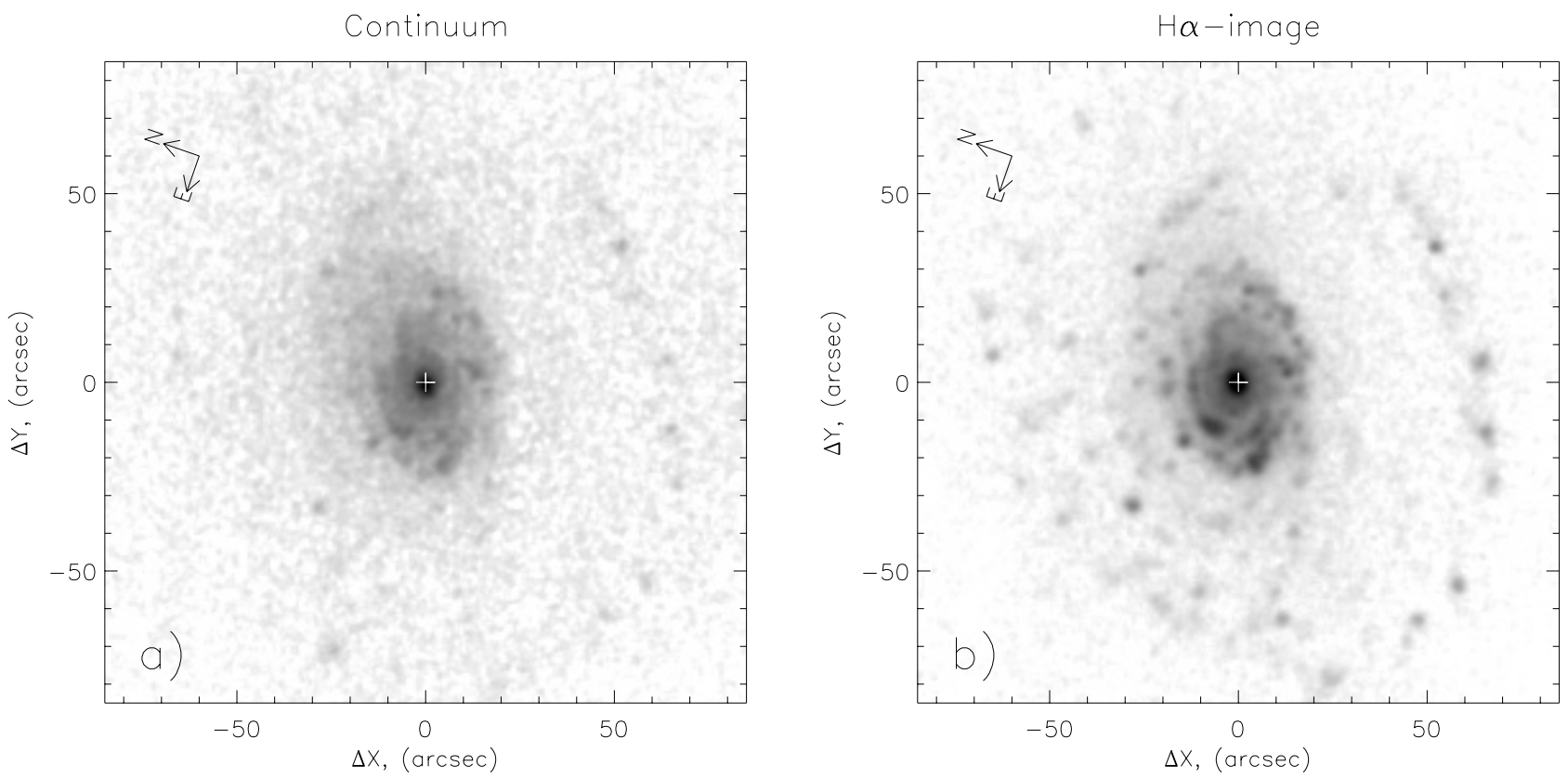

Velocities, $\mathrm{km} / \mathrm{s}$

$22002300 \quad 2400 \quad 2500 \quad 2600$

$2700 \quad 2800$
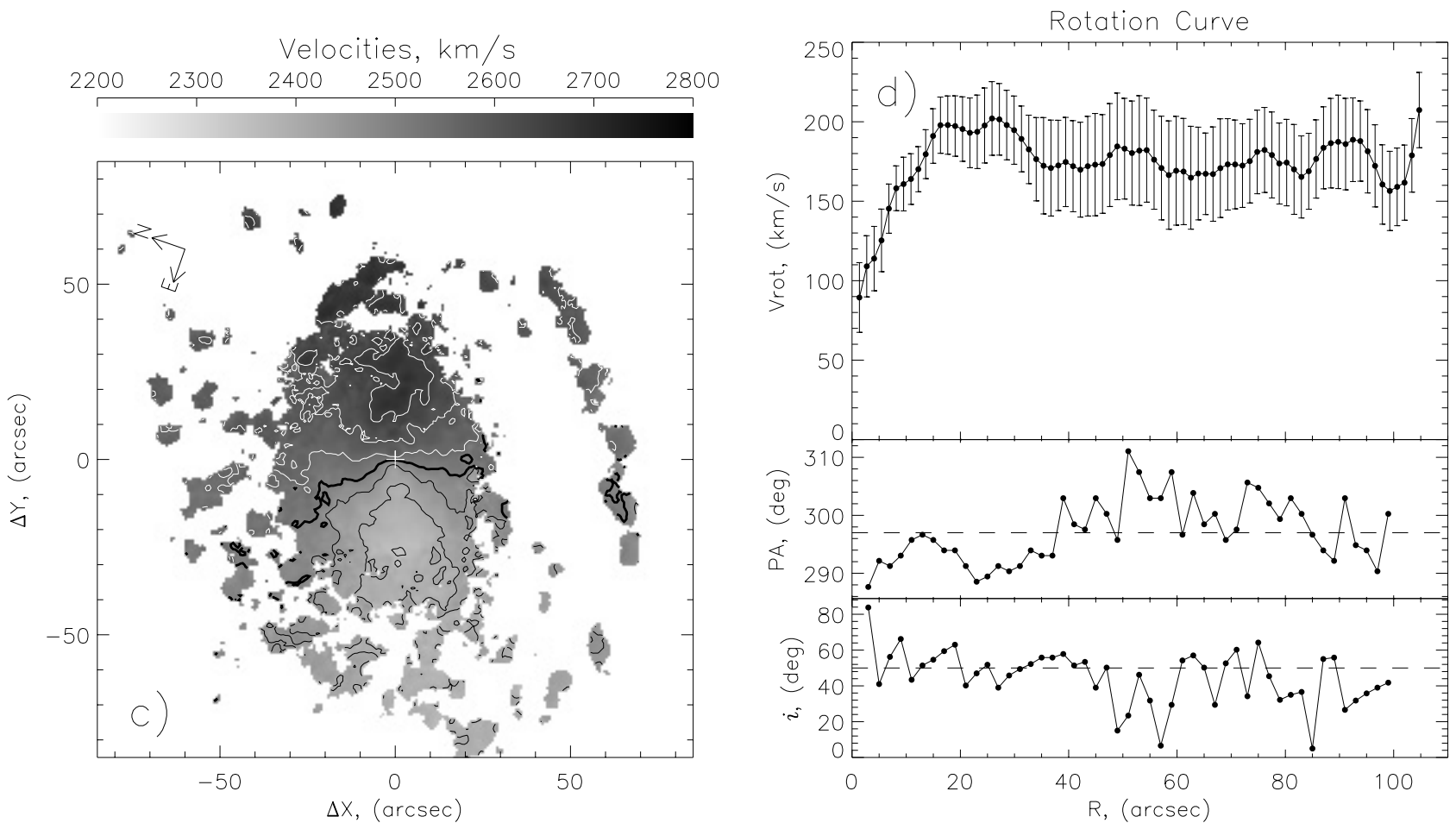

Fig. 17. The same as Fig. 5 for NGC 4814. 
A. M. Fridman et al.: The orientation parameters of spiral galaxies, Online Material p 20

\section{Galaxy: NGC5371}
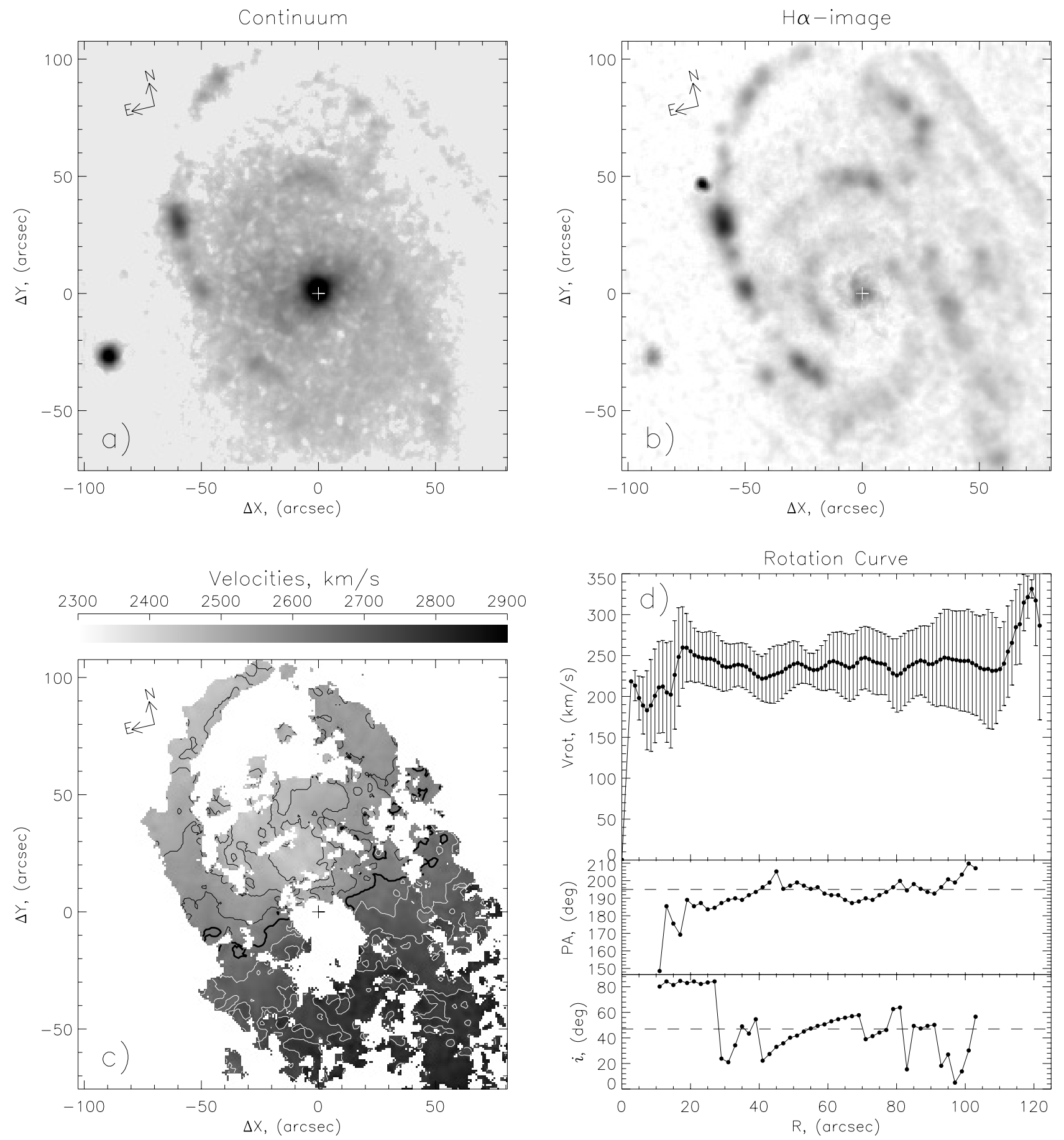

Fig. 18. The same as Fig. 5 for NGC 5371. 
A. M. Fridman et al.: The orientation parameters of spiral galaxies, Online Material p 21

\section{Galaxy: NGC6643}
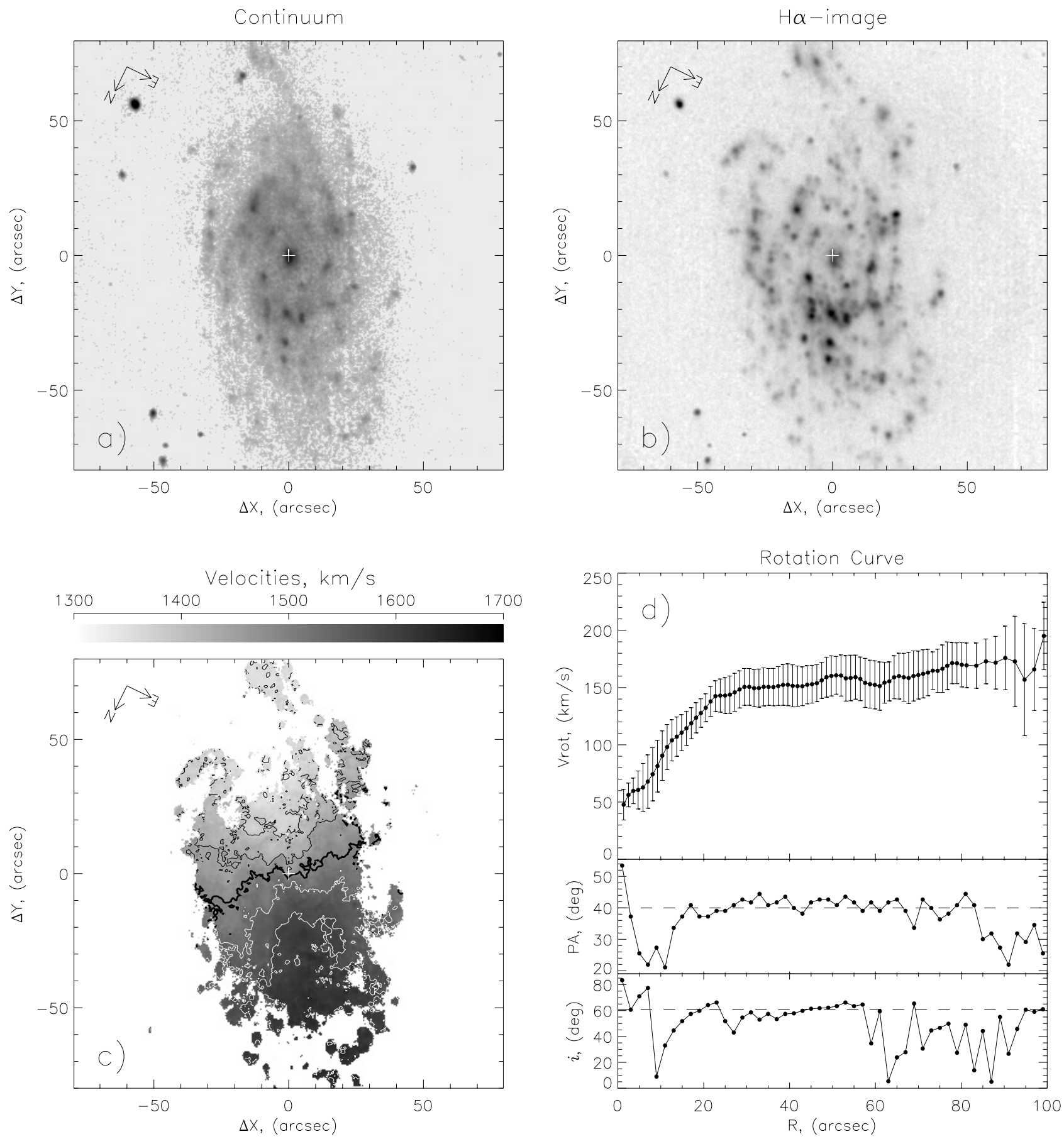

Fig. 19. The same as Fig. 5 for NGC 6643. 